\title{
Therapeutic inhibition of soluble brain TNF promotes remyelination by increasing myelin phagocytosis by microglia
}

\author{
Maria Karamita, ${ }^{1}$ Christopher Barnum, ${ }^{2}$ Wiebke Möbius, ${ }^{3}$ Malú G. Tansey, ${ }^{2}$ David E. Szymkowski, ${ }^{4}$ \\ Hans Lassmann, ${ }^{5}$ and Lesley Probert ${ }^{1}$ \\ 'Laboratory of Molecular Genetics, Hellenic Pasteur Institute, Athens, Greece. ${ }^{2}$ Department of Physiology, Emory \\ University, Atlanta, Georgia, USA. ${ }^{3}$ Department of Neurogenetics, Max Planck Institute for Experimental Medicine, \\ Goettingen, Germany. ${ }^{4}$ Xencor, Monrovia, California, USA. ${ }^{5}$ Department of Neuroimmunology, Centre for Brain Research, \\ Medical University of Vienna, Vienna, Austria.
}

Multiple sclerosis (MS) is an inflammatory CNS demyelinating disease in which remyelination largely fails. Transmembrane TNF (tmTNF) and TNF receptor 2 are important for remyelination in experimental MS models, but it is unknown whether soluble TNF (solTNF), a major proinflammatory factor, is involved in regeneration processes. Here, we investigated the specific contribution of solTNF to demyelination and remyelination in the cuprizone model. Treatment with XPro1595, a selective inhibitor of solTNF that crosses the intact blood-brain barrier (BBB), in cuprizone-fed mice did not prevent toxin-induced oligodendrocyte loss and demyelination, but it permitted profound early remyelination due to improved phagocytosis of myelin debris by CNS macrophages and prevented disease-associated decline in motor performance. The beneficial effects of XPro1595 were absent in TNF-deficient mice and replicated in tmTNF-knockin mice, showing that tmTNF is sufficient for the maintenance of myelin and neuroprotection. These findings demonstrate that solTNF inhibits remyelination and repair in a cuprizone demyelination model and suggest that local production of solTNF in the CNS might be one reason why remyelination fails in MS. These findings also suggest that disinhibition of remyelination by selective inhibitors of solTNF that cross the BBB might represent a promising approach for treatment in progressive MS.

Conflict of interest: DES is an employee of Xencor and also holds stocks and stock options in the company.

Submitted: March 8, 2016 Accepted: March 16, 2017 Published: April 20, 2017

Reference information: JCI Insight. 2017;2(8):e87455. https:// doi.org/10.1172/jici.insight.87455.

\section{Introduction}

Demyelination of the axons of CNS neurons can result from a wide range of insults, including metabolic disturbances, certain viral infections, physical injury, and chronic inflammatory diseases, particularly multiple sclerosis (MS). It is inevitably accompanied by the death of mature oligodendrocytes (OLG) and axonal damage. In counterbalance, the CNS quickly responds to demyelination by the activation of endogenous repair responses that share mechanisms of neuroinflammation, remyelination, and neuroprotection, and therefore represent important common targets for therapeutic intervention in a wide range of pathologies (1). In MS, the risk of disease development is determined partly by genetic factors relating to immune system activation $(2,3)$. In early disease, treatments that modulate immune function, particularly $\mathrm{T}$ and $\mathrm{B}$ cell responses, are effective at reducing disease relapses and preserving CNS structure and function (4) However, much less is known about how demyelination continues to expand and why remyelination largely fails in later progressive forms of MS $(5,6)$, where immune system involvement is reduced, blood-brain barrier $(\mathrm{BBB})$ function is at least partially recovered, and current antiinflammatory and immunosuppressive therapies are ineffective (7).

Remyelination is an inherent regenerative process of the adult CNS that is triggered in response to myelin damage (8). It is mediated by OLG progenitor cells (OPC) that migrate toward environmental cues, proliferate, and differentiate to synthesize new myelin sheaths around demyelinated axons (8). In progressive MS, areas of slowly expanding demyelination correlate with areas of remyelination, indicating that demyelination occurs repeatedly at the same CNS locations, thereby exposing axons to continuous injury (5). These data suggest that the mechanisms regulating demyelination and remyelination might be 
intricately linked. An understanding of why remyelination fails in MS, and the identification of mediators that promote or inhibit remyelination, are important for the development of neuroprotective strategies for the treatment of progressive MS. One potential approach is modulation of the inflammatory environment in demyelinating lesions to favor remyelination, although cellular and molecular mechanisms that are specifically involved in the remyelination process need to be elucidated. Inflammation plays a dual role in CNS demyelination; chronic inflammation promotes degeneration, while acute inflammation favors remyelination (9). As an example, blood-derived macrophages are mainly responsible for autoimmune demyelination in experimental autoimmune encephalomyelitis (EAE) $(10,11)$ but facilitate remyelination via phagocytosis of myelin debris and clearance of myelin inhibitors in a toxin-induced model $(12,13)$. Delineating the mechanisms that underlie the demyelinating versus remyelinating functions of microglia/ macrophages should lead to novel therapeutic targets.

$\mathrm{TNF}$ is a master proinflammatory product of activated microglia and peripheral macrophages that is implicated in the pathogenesis of CNS demyelination. TNF induces OLG death and myelin damage when added to mouse spinal cord cultures (14) and triggers spontaneous demyelination when overexpressed in CNS glia of transgenic mice (15). On the other hand, in an experimental model of demyelination and remyelination induced by dietary cuprizone (CPZ), TNF-deficient $\left(\operatorname{Tnf}^{\prime-}\right)$ mice demyelinate but fail to remyelinate, and they show reduced numbers of proliferating OPC in lesions, pointing to a critical role of TNF in CNS remyelination (16). Since TNF comprises, at minimum, a two-ligand (soluble and transmembrane TNF) and two-receptor (TNFR1 and TNFR2) system - with ligands and receptors being differentially expressed and regulated on different cell types (17) - identification of the TNF ligand/receptor pairs and cellular mechanisms involved in remyelination might lead to the development of novel therapeutics for the treatment of progressive MS. Previous studies showed that TNFR2 is necessary for OPC proliferation and remyelination after the removal of dietary toxin in the CPZ model (16). Further, selective inhibition of soluble TNF (solTNF) by the administration of XPro1595, a dominant-negative TNF analogue (18), but not nonselective inhibition of solTNF and transmembrane TNF (tmTNF) by etanercept, promotes remyelination in EAE (19). These findings, together with recent evidence that oligodendroglial TNFR2 is sufficient for TNF-mediated remyelination in EAE (20), provide strong evidence that tmTNF promotes remyelination via TNFR2 present on oligodendroglial cells. However, it is not clear whether solTNF inhibits the remyelination process directly or indirectly by increasing neuroinflammation. In this study, we exploited our finding that XPro1595 can penetrate the intact brain, using it to investigate the role of solTNF in a CPZ model. We show that therapeutic inhibition of solTNF in the brain by XPro1595 does not prevent OLG loss or demyelination, but permits early myelin repair and preservation of axons by enhancing the phagocytosis of myelin debris in demyelinated lesions by CNS macrophages. Studies in Tnf ${ }^{\prime-}$ and tmTNFknockin $\left(\operatorname{tm} \operatorname{Tn}^{f^{/ 4}}\right)$ mice further show that tmTNF is necessary for the beneficial effects of XPro1595. Collectively, our data demonstrate opposing effects of solTNF and tmTNF in inhibiting and promoting CNS remyelination, respectively, and suggest that selective inhibition of solTNF by brain-penetrating agents such as XPro1595 represents a promising approach for enhancing endogenous CNS repair mechanisms in progressive MS.

\section{Results}

CPZ-induced demyelination is a well-characterized and highly reproducible experimental model for studying mechanisms of CNS demyelination and remyelination in mice of susceptible strains, including C57BL/6 (B6) (21). CPZ is a copper chelator, and CPZ feeding induces copper deficiency, the selective death of OLG, and CNS demyelination that is readily measurable in the corpus callosum (22). Demyelination is associated with CNS-compartmentalized neuroinflammation that develops behind an intact BBB (23), although blood-derived neutrophils also play critical roles in early disease (24). CPZ administration for 6 weeks in young-adult B6 mice induces acute demyelination followed by almost-complete remyelination in the weeks after CPZ withdrawal (21), while administration for 12 weeks or more causes chronic demyelination and irreversible axonal damage (25). The acute model is particularly useful for studying OPC responses to demyelination and remyelination, as well as key aspects of progressive MS such as oxidative damage and mitochondrial injury in the presence of chronically activated microglia (7).

Local inhibition of solTNF in the brain promotes myelin recovery after CPZ-induced demyelination. Acute demyelination was induced in WT B6 mice as described (21). In this model, demyelination shows a highly reproducible time course of pathological and gene expression changes $(21,22,26,27)$. Severe demyelination 


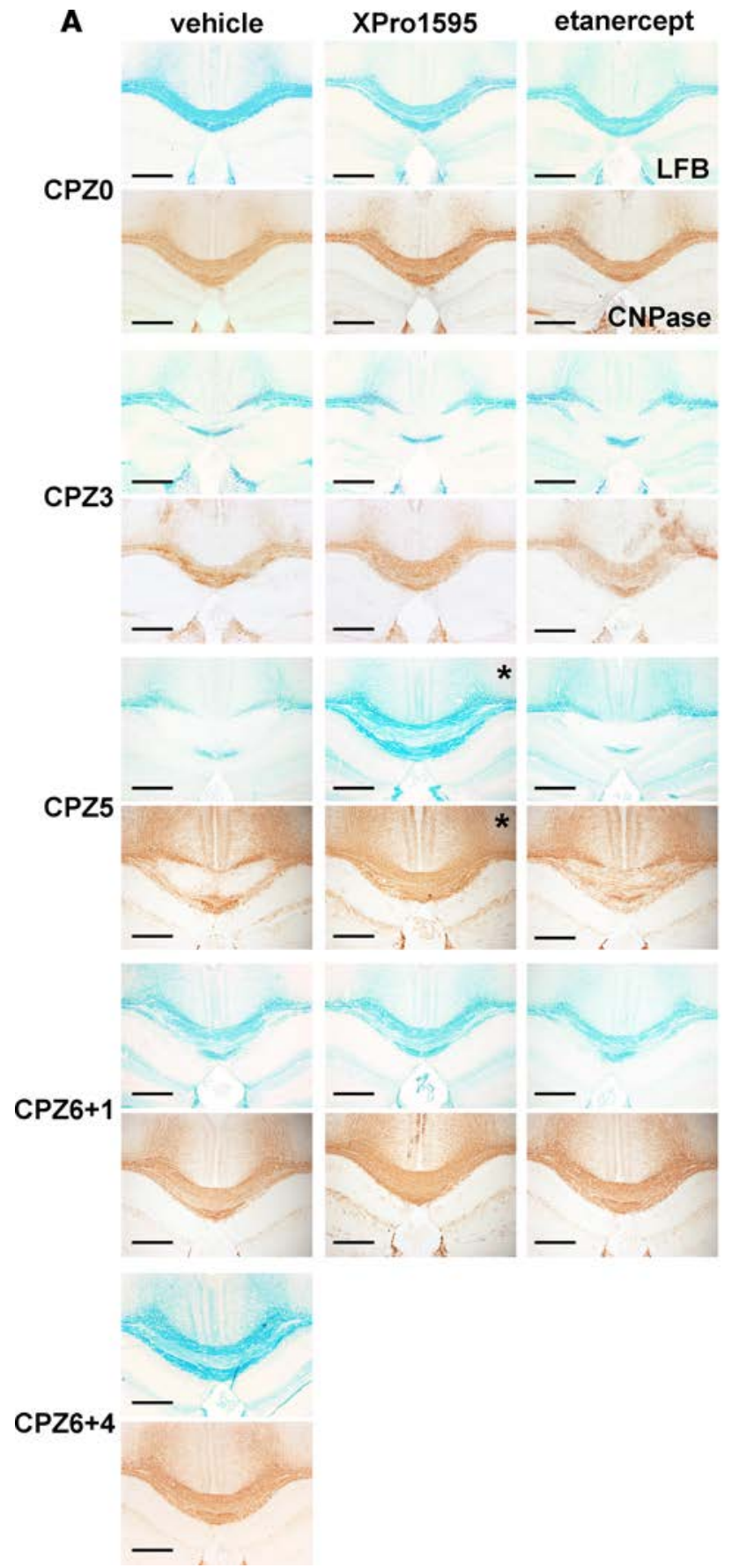

B

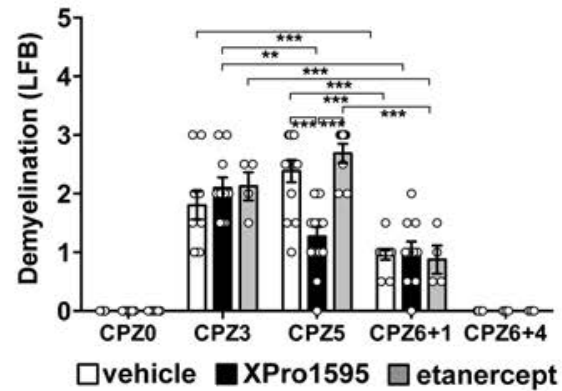

C

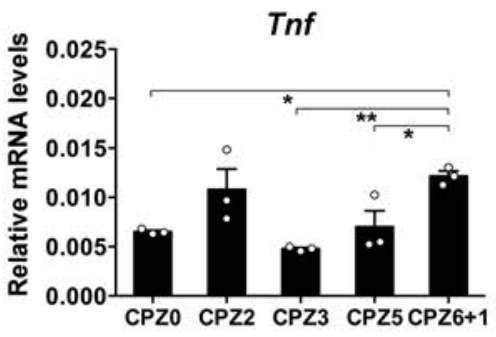

D

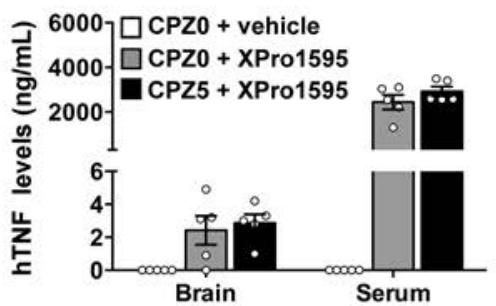

$\mathbf{E}$

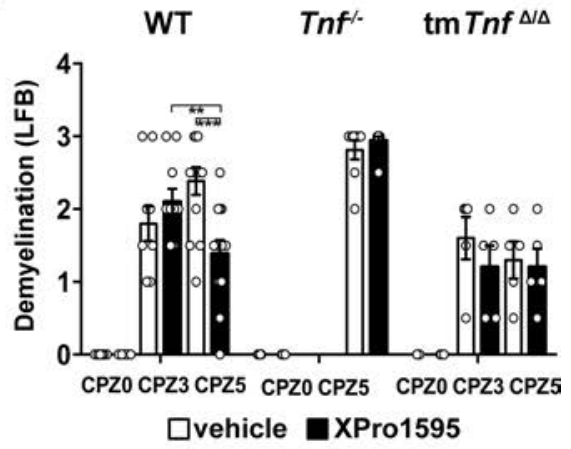

Figure 1. Therapeutic inhibition of soluble TNF in brain does not alter cuprizone-induced demyelination but promotes myelin recovery in a transmembrane TNF-dependent manner. (A) Klüver-Barrera Luxol fast blue staining (LFB) and 2', 3'-cyclic nucleotide 3'-phosphodiesterase (CNPase) immunostaining of myelin in serial brain coronal paraffin sections from representative vehicle- (left column), XPro1595- (middle column), and etanercept-treated (right column) naive mice (CPZO) or cuprizone-fed (CPZ-fed) CPZ3, CPZ5, CPZ6+1, and CPZ6+4 mice ( $n=8$ for vehicle-treated CPZO, $n=4$ for XPro1595-treated CPZO, and $n=6-12$ for all other time points). Scale bars: $500 \mu \mathrm{M}$. (B) Semiquantitative scoring of demyelination (loss of LFB staining) in the callosum of the same groups of mice represented in A. (C) Levels of Tnf mRNA transcripts relative to Gusb in whole brain samples from vehicle-treated CPZO and CPZ2, CPZ3, CPZ5, and CPZ6+1 mice by quantitative PCR ( $n=4$ per group). (D) Human TNF levels in brain and serum from vehicle- and XPro1595-treated CPZO and XPro1595-treated CPZ5 mice using a human TNF immunoassay ( $n=5$ per group). (E) Semiquantitative scoring of demyelination (loss of LFB staining) in coronal paraffin sections of corpus callosum of vehicle- and XPro1595-treated CPZO, CPZ3, and CPZ5 WT, Tnf ${ }^{-1}$, or tmTnf $\mathrm{fm}^{4 / 4} \mathrm{mice}^{(}(n=6-8$ per group). The results shown are from one representative ([E] Tnf $\left.f^{-/}, \operatorname{tmTnf}^{4 / 4}\right)$ of two $(\mathbf{C}$ and $\mathbf{D})$ or three ([A, B, and E] WT) independent experiments. Statistical significance after comparisons between measurements of demyelination in the different mouse strains (B and E) and mRNA levels (C) by two-way ANOVA with Bonferroni's test and ordinary one-way ANOVA with Tukey's test, respectively, is shown. ${ }^{*} P<0.05,{ }^{* *} P<0.01,{ }^{* * *} P<0.001$. (B and E) Comparisons between measurements of demyelination in CPZO mice with other groups are all significant and not annotated on the plots. (A) Asterisks show early recovery of myelin. (B, C, and $\mathbf{E}$ ) Circles show values for individual mice. 
occurred in the corpus callosum after both 3 (CPZ3) and 5 (CPZ5) weeks of CPZ feeding, compared with naive (CPZ0) controls, as shown by Klüver-Barrera Luxol fast blue staining (LFB) (Figure 1A and Figure 1B) and immunostaining for 2', 3'-cyclic 3'-phosphodiesterase (CNPase) (Figure 1A and Supplemental Figure 1A; supplemental material available online with this article; https://doi.org/10.1172/jci.insight.87455DS1). The corpus callosum in CPZ-fed vehicle-treated WT mice showed an increase in total volume at both CPZ3 $\left(2.56 \pm 0.08 \mathrm{~mm}^{3}\right)$ and CPZ5 $\left(2.82 \pm 0.03 \mathrm{~mm}^{3}\right)$ compared with CPZ0 $\left(2.25 \pm 0.04 \mathrm{~mm}^{3}\right)$, despite significant demyelination (Supplemental Figure 2A), possibly as a combined result of reduced myelin compaction and increased numbers of inflammatory cells (see below). Adjustment of LFB measurements at each experimental time point to incorporate these differences showed similar results (Supplemental Figure 2B). CPZ removal from the diet at week $6(\mathrm{CPZ6})$ was followed by progressive recovery of myelin as measured after $1(\mathrm{CPZ6}+1)$ and $4(\mathrm{CPZ6}+4)$ weeks (Figure 1A, Figure 1B, and Supplemental Figure 1A). In parallel with the histopathological changes, and as reported in $\mathrm{B} 6$ mice $(26,27)$, levels of mRNA encoding the structural protein myelin basic protein (MBP) in total brain were dramatically reduced at the CPZ2, CPZ3, and CPZ5 time points and recovered again following CPZ removal $(\mathrm{CPZ6}+1)($ Supplemental Figure 1B).

Using genetic models, TNF was previously shown to advance the onset of CPZ-induced demyelination and OLG death, and to promote the remyelination process after withdrawal of dietary CPZ (16), suggesting that it may have different effects at different stages of pathology. Here, we found that brain TNF mRNA levels consistently showed a small, nonsignificant increase at CPZ2 and a second increase at CPZ6+1, when remyelination is occurring in WT mice (Figure 1C). To investigate possible differential roles of solTNF and tmTNF during the development of demyelination and remyelination, we administered XPro1595 systemically to WT, $T n f^{\prime-}$, or tm $T n f^{1 / 4}$ mice. XPro1595 is a TNF inhibitor designed by mutating key amino acids in human solTNF to eliminate binding to TNFR1 and TNFR2. Similarly to the genetic concept of dominant-negative inhibitors, XPro1595 exchanges subunits with and thus destroys the native homotrimeric solTNF ligand, while fully preserving tmTNF activity $(18,28)$. XPro1595 is equally inhibitory against mouse solTNF and equally protective of mouse tmTNF, as it is for human TNF (28). We treated WT mice using a therapeutic protocol whereby XPro1595 was administered twice-weekly starting from the end of the second week of feeding (at the onset of first histopathological changes), and we sacrificed mice at different time points during and after CPZ feeding. XPro1595 administration did not alter initial demyelination, which was equal to that in vehicle-treated controls at CPZ3 (Figure 1A, Figure 1B, and Supplemental Figure 1A). However, XPro1595 stimulated the early recovery of myelin at CPZ5 compared with vehicle controls, a time point where CPZ was still present in the diet (Figure 1A, Figure 1B, and Supplemental Figure 1A). The corpus callosum volume in XPro1595-treated WT mice showed an equal increase to that in vehicle-treated controls at both CPZ3 $\left(2.58 \pm 0.02 \mathrm{~mm}^{3}\right)$ and CPZ5 $\left(2.87 \pm 0.09 \mathrm{~mm}^{3}\right)$ compared with CPZ0 $\left(2.33 \pm 0.03 \mathrm{~mm}^{3}\right)$ (Supplemental Figure 2A). Adjustment of LFB measurements at each experimental time point to incorporate these differences in XPro1595-treated mice revealed similar results (Supplemental Figure 2B).

Since CPZ demyelination is not associated with BBB damage (23), we checked whether peripherally administered XPro1595 reached the brain. We measured levels of XPro1595 in serum and brain homogenates from CPZ0 and CPZ-treated mice that were treated with XPro1595 or saline for 3 weeks prior to sacrifice at CPZ5 (6 injections) using an antibody specific for human TNF, which recognizes the human dominant negative TNF protein epitopes in XPro1595 but not mouse TNF. No XPro1595 signal was detectable in brains from vehicle-treated naive mice (Figure 1D). In contrast, equal levels of XPro1595 were measured in brains from XPro1595-treated naive and CPZ-fed mice, and were in the range of $400 \mathrm{pg} /$ $\mathrm{ml}$ (PBS-perfused mice) (Supplemental Figure 3) to 3,000 pg/ml (nonperfused mice) (Figure 1D). Levels of XPro1595 in the sera from XPro1595-treated mice were more than 1,000-fold higher than levels detected in the CNS on the day of sacrifice, suggesting an inefficient yet still pharmacologically relevant flux across the BBB. As pathological levels of TNF in the CNS have been reported to be in the picogram per $\mathrm{ml}$ range (29), the measured levels of XPro1595 are predicted to inhibit $>99 \%$ of solTNF in the brain (18). These results show that XPro1595 penetrates and accumulates in the brain at pharmacologically relevant levels.

To exclude the participation of peripherally derived TNF in the effects observed, we treated mice by twice-weekly s.c. injection of etanercept $(10 \mathrm{mg} / \mathrm{kg})$, a nonselective inhibitor that blocks both solTNF and tmTNF (30), according to our therapeutic protocol. Etanercept is a human TNFR2-Fc (IgG1) fusion protein that blocks both solTNF and tmTNF and minimally penetrates the BBB (31). Etanercept did not prevent initial demyelination, which was equal to that in XPro1595- and vehicle-treated mice at CPZ3, nor did 

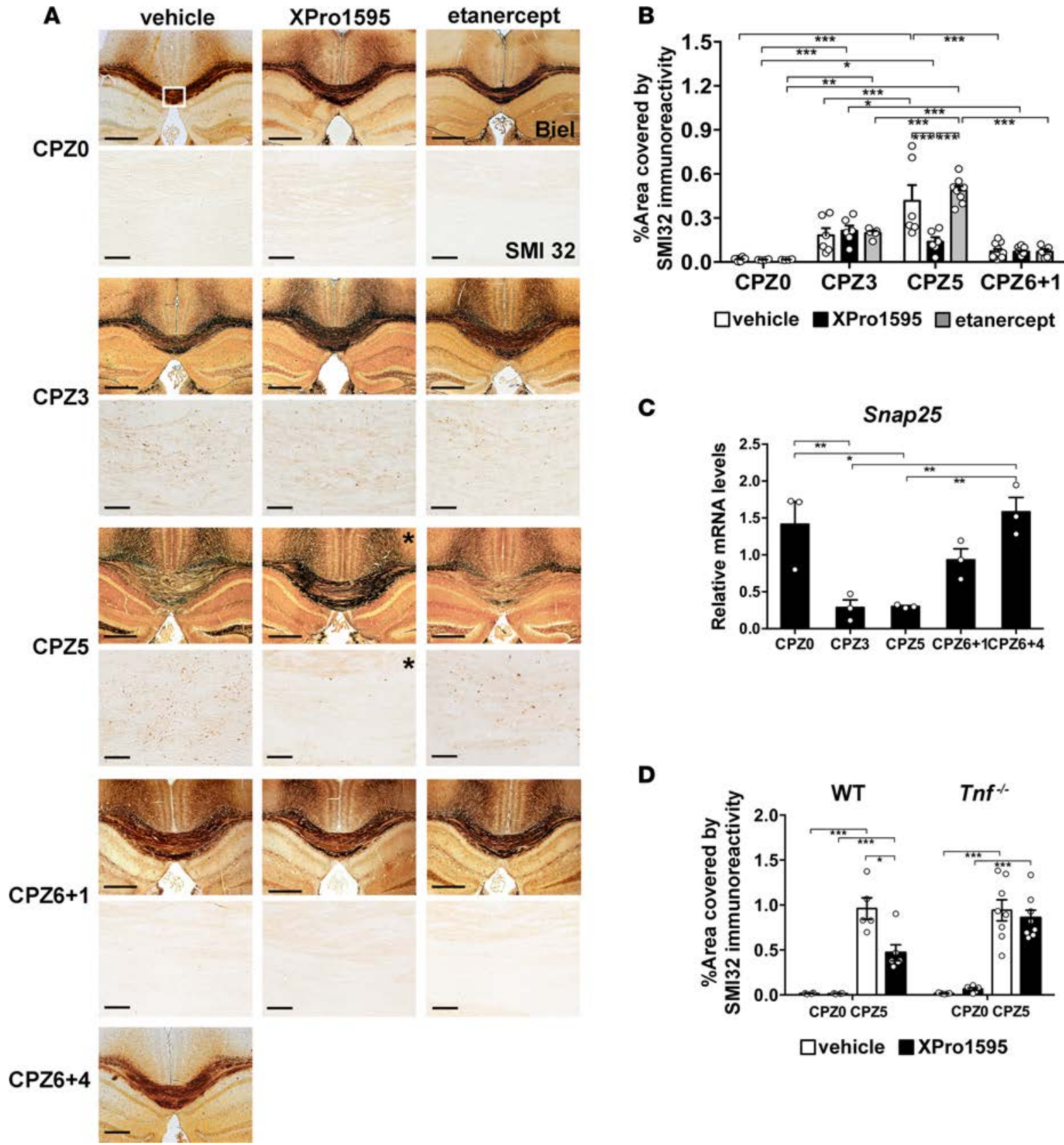

C

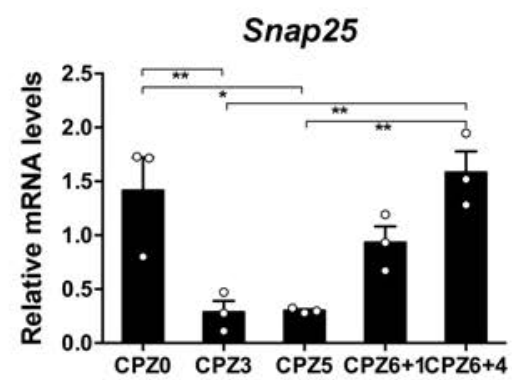

D

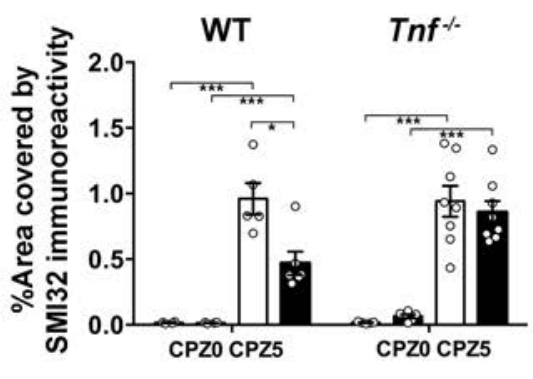

$\square$ vehicle $\square$ XPro1595

Figure 2. Therapeutic inhibition of soluble TNF in brain does not alter acute cuprizone-induced axon damage but prevents its progression in the corpus callosum in a TNF-dependent manner. (A) Bielschowsky's silver staining (Biel) and neurofilament $\mathrm{H}$ nonphosphorylated (SMI 32) immunostaining of axons in brain coronal sections from representative vehicle- (left column), XPro1595- (middle column), and etanercept-treated (right column) naive mice (CPZO) or cuprizonefed (CPZ-fed) CPZ3, CPZ5, CPZ6+1, and CPZ6 +4 mice ( $n=8$ for vehicle-treated CPZO, $n=4$ for XPro1595-treated CPZO, and $n=6-12$ for other time points). The area used for quantitating axon damage is shown in the vehicle-treated Bielschowsky-stained CPZO sample. Scale bars: $500 \mu M$ for Bielschowsky, $50 \mu M$ for SMI 32. (B) Quantitative representation of SMI 32 immunoreactivity in the corpus callosum of groups of mice represented in A. (C) Levels of Snap25 mRNA transcripts relative to Gusb in whole brain samples from CPZO, CPZ3, CPZ5, CPZ6 +1 , and CPZ6+4 mice by quantitative PCR ( $n=4$ per group). (D) Quantitative representation of SMI 32 immunoreactivity in the corpus callosum of vehicle- and XPro1595-treated CPZO and CPZ5 Tnf ${ }^{-1-}$ or WT mice ( $n=6-8$ per group). The results shown are from one representative ([D] Tnf $f^{\prime-}$ ) of two (C) or three ([A, B, and D] WT) independent experiments. Statistical significance after comparisons between measurements of immunoreactivity in the different mouse strains (B, D) and mRNA levels (C) by two-way ANOVA with Bonferroni's test and ordinary one-way ANOVA with Tukey's test, respectively, is shown. ${ }^{*} P<0.05$, ${ }^{*} P<0.01,{ }^{* *} P<0.001$. (A) Asterisks show maintenance of axon integrity. (B-D) Circles show values for individual mice.

it alter the subsequent recovery of myelin at CPZ5 (Figure 1A, Figure 1B, and Supplemental Figure 1A). This result shows that peripherally derived TNF does not contribute to demyelination or remyelination in the CPZ model and indicates that XPro1595 promotes myelin recovery by inhibiting solTNF locally in the CNS. To verify that peripherally administered etanercept has limited brain penetrance, we measured 
levels of etanercept in serum and brain homogenates from CPZ0 and CPZ-fed mice that were treated with etanercept or saline for 3 weeks prior to sacrifice at CPZ5 (6 injections) using an immunoassay specific for human TNFR2 that does not cross-react with mouse TNFR2. Low levels of etanercept, approximately 100 times lower than those of XPro1595, were detectable in brain homogenates from PBS-perfused etanercepttreated naive or CPZ-fed (2-3 pg/ml) mice but not from vehicle-treated mice (Supplemental Figure 3). These results show that etanercept fails to accumulate at significant levels in the brain of naive or CPZtreated animals.

Given that TNF and TNFR2, which is activated by tmTNF (32), are reported to play a role in OLG and myelin regeneration (16), we first checked whether the increased recovery of myelin in XPro1595treated animals was dependent on TNF, and therefore by deduction tmTNF, by administering XPro1595 to CPZ-fed $\mathrm{Tnf}^{\prime-}$ mice. Vehicle-treated $\mathrm{Tnf}^{\prime-}$ mice lacking both solTNF and tmTNF developed robust and equal demyelination at CPZ5, similar to that in vehicle-treated WT mice (Figure 1E). Unlike WT mice, however, XPro1595-treated $\mathrm{Tnf}^{1-}$ mice did not show myelin recovery at this time point, suggesting that tmTNF is necessary for the myelin recovery seen in XPro1595-treated WT mice (Figure 1E). To confirm a role for tmTNF in the maintenance of myelin, we next administered XPro1595 to CPZ-fed tm TNF ${ }^{\Delta / 4}$ mice, which carry an uncleavable TNF allele and constitutively produce functional, normally regulated tmTNF without solTNF (33). Naive (CPZ0) vehicle- and XPro1595-treated tm $T N F^{4 / \Delta}$ mice showed normal myelination, similar to that in WT and $T n f^{\prime-}$ mice, as determined by LFB staining. CPZ-fed tm TNF ${ }^{4 / 4}$ mice developed demyelination in response to CPZ feeding, similar to that in WT mice at CPZ3, but demyelination did not progress further at CPZ5, as it did in WT and $\mathrm{Tnf}^{\prime-}$ mice (Figure 1E). As expected, XPro1595

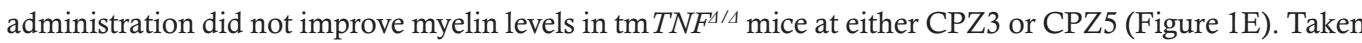
together, these results reveal a previously unknown role for solTNF in inhibiting myelin repair, as shown by early myelin recovery following XPro1595 treatment in WT mice. They also reveal that tmTNF is necessary for early remyelination in XPro1595-treated WT mice, as shown by the failure of myelin recovery in XPro1595-treated $\mathrm{Tnf}^{\prime-}$ mice, and maintenance of myelin integrity in $\mathrm{tm}^{\mathrm{T}} \mathrm{TN} \mathrm{F}^{\mathrm{s} / \Delta}$ mice, at CPZ5.

Inhibition of brain solTNF reduces CPZ-induced axonal damage. Although neuronal pathology is not wellstudied in the CPZ model, demyelination is associated with axon damage, and its severity increases with age (34) and duration of treatment (25). Consistent with this, CPZ feeding in vehicle-treated mice induced progressive axon damage in the corpus callosum, as monitored at CPZ3 and CPZ5 by a reduction of Bielschowsky's Biel silver-stained axons (Figure 2A) and an increase in area covered by SMI 32 immunostaining for nonphosphorylated neurofilament $\mathrm{H}$ (Figure 2B). Adjustment of SMI 32 immunostaining results at CPZ0, CPZ3, and CPZ5 to incorporate differences in corpus callosum volume between groups showed similar results (Supplemental Figure 2C). Axon damage was almost completely absent by 1 week after CPZ withdrawal (Figure 2A and Figure 2B), showing that axon damage in this acute model is reversible. Levels of mRNA encoding the synaptic protein Snap25 in total brain were reduced at the CPZ3 and CPZ5 time points and progressively recovered again after $\mathrm{CPZ}$ withdrawal (CPZ6+1, CPZ6+4) (Figure 2C).

Inhibition of solTNF by XPro1595 treatment did not prevent initial axon damage measured at CPZ3, but it prevented the further progression of damage at CPZ5 in the vehicle-treated group (Figure 2A, Figure 2B, and Supplemental Figure 2C). Etanercept treatment had no effect on axonal damage at any time point, as monitored by Biel silver staining (Figure 2A) or SMI 32 immunostaining (Figure 2A and Figure 2B), suggesting that peripherally derived TNF does not contribute to axon damage in the CPZ model. These results show that local inhibition of solTNF in the brain by XPro1595 prevents the progressive accumulation of axon damage in the corpus callosum during $\mathrm{CPZ}$ administration.

tmTNF and its two receptors, TNFR1 and TNFR2, are known to be important for neuroprotection (17, 35). Therefore, we investigated whether protection against axonal damage in XPro1595-treated animals is dependent on TNF, and therefore by tmTNF, by administering XPro1595 to CPZ-fed Tnf ${ }^{\prime-}$ mice. As in WT mice, vehicle-treated $\mathrm{Tnf}^{\prime-}$ mice lacking both solTNF and tmTNF developed axonal damage, as measured by SMI 32 immunoreactivity, at CPZ5 (Figure 2D). Unlike in WT mice, where XPro1595 treatment prevented further axon damage at this time point, $\mathrm{Tnf}^{\text {/- }}$ mice treated with XPro1595 showed equal axonal damage as vehicle-treated controls (Figure 2D). Taken together, these results show that local inhibition of solTNF in the brain protects against progressive CPZ-induced axonal damage. In addition, our observations that axonal damage fully develops in Tnf ${ }^{\prime-}$ mice, which lack both solTNF and tmTNF, and that XPro1595-mediated protection is abrogated by TNF deficiency, support a role for tmTNF in axon protection, most likely as an indirect result of its positive effects on myelin. 


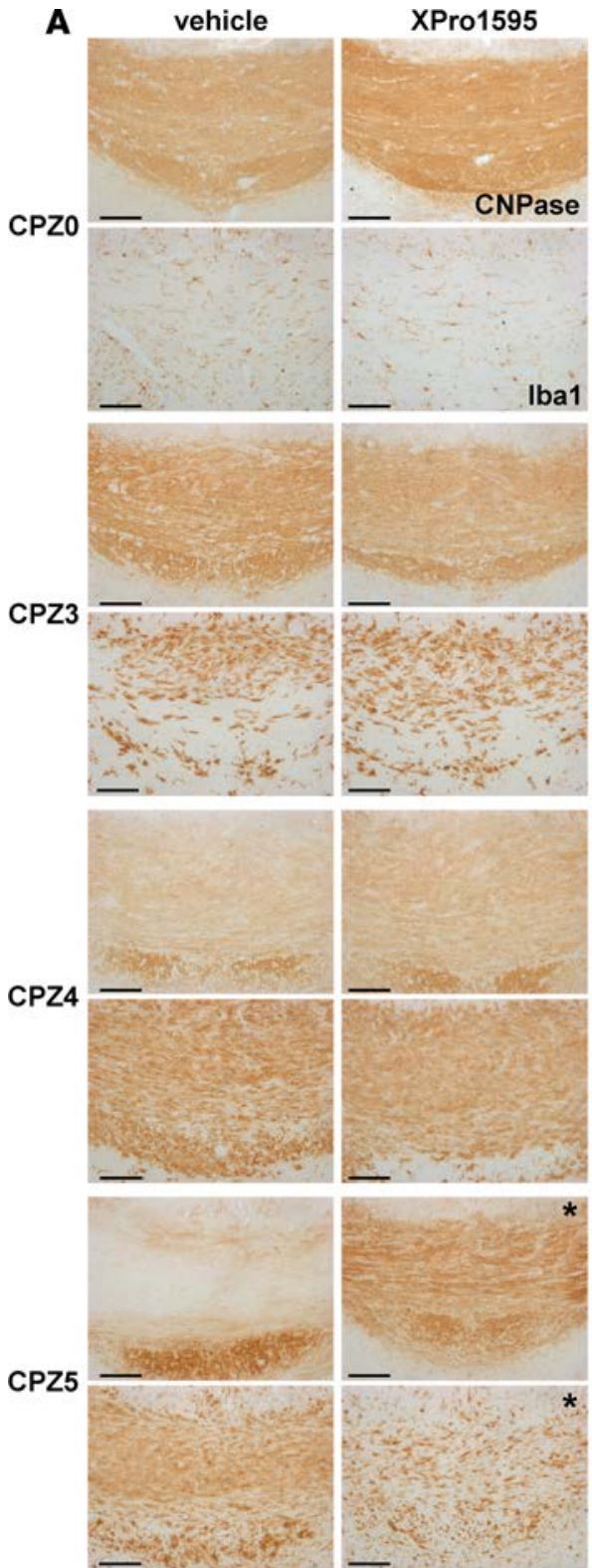

B

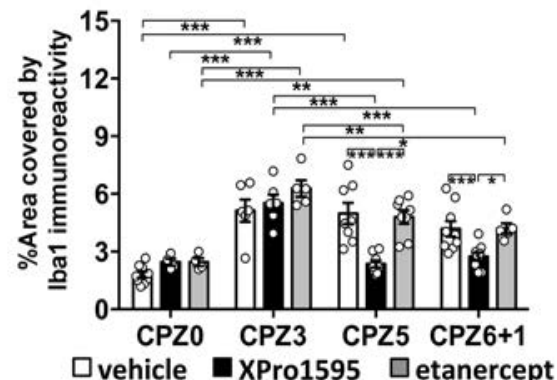

C

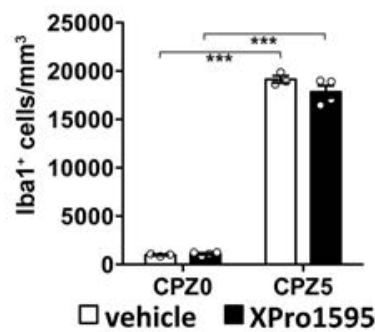

D

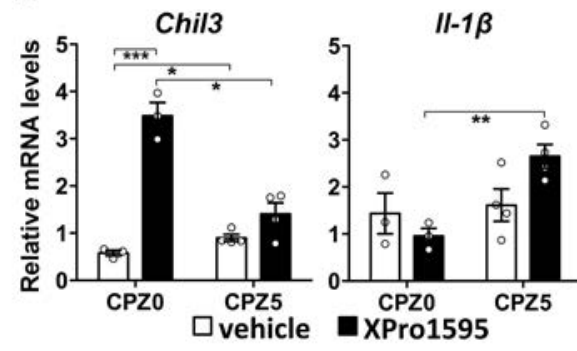

$\mathbf{E}$



Figure 3. Therapeutic inhibition of soluble TNF in brain promotes early resolution of microgliosis in demyelinated lesions. (A) Immunostaining for lonized calciumbinding adaptor molecule 1 (Iba1) and 2', 3'-cyclic nucleotide 3'-phosphodiesterase (CNPase) in serial coronal paraffin sections of corpus callosum from representative vehicle- (left column) and XPro1595-treated (right column) CPZO, CPZ3, CPZ4, and CPZ5 mice ( $n=8$ for vehicle-treated CPZO, $n=4$ for XPro1595-treated CPZO, and $n=6-12$ for all other time points). Scale bars: $100 \mu \mathrm{M}$. (B) Quantitative representation of area covered by lba1 immunoreactivity in the callosum in groups of mice represented in A. (C) Numbers of Iba1-immunoreactive microglia/ $\mathrm{mm}^{3}$, as measured by stereology in brain coronal cryostat sections through callosum from representative vehicle- and XPro1595-treated CPZO and CPZ5 mice ( $n=3-4$ for each group). (D) Levels of Chil3 and II-1 $\beta$ mRNA transcripts relative to Gapdh in whole brain samples from vehicle- and XPro1595-treated CPZO and CPZ5 mice by quantitative PCR ( $n$ = 3-4 per group). (E) Quantitative representation of area covered by lba1 immunoreactivity in coronal paraffin sections of corpus callosum of vehicle- and XPro1595-treated CPZO and CPZ5 Tnf $^{-1-}$ or WT mice ( $n=6-8$ per group). The results shown are from one representative ([C, D, and $\mathbf{E}] \mathrm{Tnf}^{-/-}$) of three ([A, B, and E] WT) independent experiments. Statistical significance after comparisons between areas of Iba1 immunoreactivity in the different mouse strains (B and $\mathbf{E}$ ), numbers of Iba1-immunoreactive cells (C), and brain mRNA levels (D) by two-way ANOVA with Bonferroni's test, is shown. ${ }^{*} P<0.05$, ${ }^{*} P<0.01,{ }^{* *} P<0.001$. (A) Asterisks show early resolution of microgliosis accompanying recovery of myelin. (B, C, D, and E) Circles show values for individual mice.

Inhibition of solTNF in the brain promotes early resolution of microgliosis in demyelinated lesions. CPZ feeding in vehicle-treated mice was associated with microglia activation at CPZ3 and full-blown confluent microgliosis at CPZ4 and CPZ5 in the demyelinating callosum, as measured by area covered by immunostaining for ionized calcium-binding adaptor molecule 1 (Iba1) (Figure 3, A and B, and Supplemental Figure 4A) and numbers of Iba1-immunostained cells by stereology (Figure 3C), consistent with previous findings (36). CPZ feeding in vehicle-treated mice was also associated with astrocyte activation at CPZ5 in the corpus callosum, as measured by area covered by glial fibrillary acidic protein (GFAP) immunostaining (Supplemental Figure 2D). In parallel with these pathological changes, and as reported in B6 mice (26), brain levels of mRNAs encoding inflammatory cytokines and mediators, including main products of activated glia such as TNF (Figure 1C), CCL2, and CXCL16, were increased during CPZ feeding with two peaks of expression: one after the initiation of $\mathrm{CPZ}$ feeding and another at $\mathrm{CPZ} 6+1$, when remyelination is occurring (Supplemental Figure 4, B and C).

XPro1595 treatment in CPZ-fed mice did not prevent early microglia activation at CPZ3 or confluent microgliosis at CPZ4, but it stimulated early resolution of microgliosis at CPZ5 and CPZ6+1, as measured by reduced areas of Iba1 immunoreactivity in the corpus callosum (Figure 3, A and B, and 
Supplemental Figure 4A, middle column, asterisks), without altering numbers of Iba1-immunoreactive cells - as measured by stereology (Figure 3C) - compared with the vehicle-treated groups. Interestingly, early resolution of microgliosis in XPro1595-treated mice correlated with early recovery of myelin at CPZ5 (Figure 1A, middle column and Figure 3A, right column). In contrast, XPro1595 treatment did not alter astrocyte activation at CPZ5, which was equal to that in vehicle-treated CPZ5 mice (Supplemental Figure 2D).

Measurement of mRNA transcripts for Chil3 (Ym1), a chitinase-like 3 protein that is associated with host defense (37) and used as a marker for protective alternatively activated macrophages, showed low-level brain expression in vehicle-treated mice at CPZ0 that was upregulated at CPZ5 (Figure 3D). Conversely, XPro1595 treatment induced marked upregulation of Chil3 in naive mice at CPZ0 that was downregulated to levels equal to those in vehicle-treated mice at CPZ5 (Figure 3D). In contrast, mRNA levels for brain IL-1 $\beta$, a cytokine important for remyelination (38) and a marker for proinflammatory macrophages, were not altered by dietary CPZ in vehicle-treated mice at CPZ0 or CPZ5 but were upregulated by XPro1595 treatment in CPZ5 mice (Figure 3D). Brain mRNA levels for other markers of proinflammatory and alternatively activated macrophages — Nos2 (iNos) and Arg-1, respectively — were not altered by dietary CPZ or XPro1595 treatment (data not shown). Etanercept treatment had no effect on the area of callosum covered by Iba1 immunoreactivity in naive or CPZ-fed animals compared with the vehicle-treated group (Figure 3B and Supplemental Figure 4A right column).

The finding that selective inhibition of solTNF with XPro1595 regulated microglia responses in the CPZ-induced demyelination model is consistent with the already known proinflammatory roles of this cytokine in the CNS (17). To investigate a possible role for tmTNF in CPZ-induced microglia reactivity, we also administered XPro1595 to CPZ-fed Tnf ${ }^{\prime-}$ mice. Unlike in WT mice, where XPro1595 treatment resolved microgliosis at CPZ5 as measured by area covered by Iba1 immunostaining, $\mathrm{Tnf}^{\prime-}$ mice treated with XPro1595 showed equal microgliosis as vehicle-treated controls (Figure 3E). Taken together, these results suggest that local inhibition of solTNF in the brain markedly regulates the phenotype of microglia, priming them toward an alternatively activated phenotype in naive mice and markedly reducing microgliosis at CPZ5, a time point at which XPro1595 treatment has profoundly promoted myelin recovery. In addition, the observations that full-blown microgliosis accompanies CPZ-induced demyelination at CPZ5 in XPro1595-treated $\mathrm{Tnf}^{\prime-}$ mice, which lack both solTNF and tmTNF, strongly support an antiinflammatory effect of tmTNF, possibly as an indirect result of its positive effects on myelination and neuroprotection.

Inhibition of solTNF in brain increases oligodendrocyte progenitor cell numbers in demyelinated lesions. Demyelination in the callosum of vehicle-treated CPZ-fed mice was associated with a robust OPC response, as measured by increased numbers of PDGFR $\alpha$-immunoreactive OPC at all time points (Figure 4, A and B), and increased area covered by PDGFR $\alpha$ immunoreactivity at CPZ3 (Supplemental Figure 5A), compared with naive animals. The response was associated with increased OPC proliferation, as shown by the increased proportions of OPC immunostained for platelet-derived growth factor receptor alpha (PDGFR $\alpha$ ) cells double-stained for Ki67 protein in the callosum at CPZ3 and CPZ5 compared with CPZ0 (Figure 4C). In the CPZ model used here, OPC start differentiating at CPZ5 to become mature, myelinating OLG by CPZ6 in WT mice $(22,39)$. This time course was reflected by increased levels of Olig2 mRNA transcripts at CPZ5 (Figure 4D) and Mbp mRNA transcripts at CPZ6+1 (Supplemental Figure 1B), recovery of myelin measured at CPZ6+1 and CPZ6+4 (Figure 1, A and B), and a drop in OPC numbers at CPZ6+1 compared with CPZ5 (Figure 4B), possibly due to OPC differentiation.

Demyelination in the callosum of XPro1595-treated mice was also associated with a robust OPC response, measured by increased numbers of PDGFR $\alpha$-immunoreactive OPC (Figure 4, A and B), and increased area covered by PDGFR $\alpha$ immunoreactivity at CPZ3 and CPZ5 (Supplemental Figures 2E and 5A), compared with naive animals. In CPZ3 lesions, the OPC response was equal but, at CPZ5, was increased compared with vehicle- and etanercept-treated mice (Figure 4, B and E, and Supplemental Figure 5A). OPC proliferation increased in XPro1595-treated CPZ-fed mice equally to that in vehicle controls (Figure 4C). To investigate a possible role for tmTNF in CPZ-induced OPC responses, we administered XPro1595 to CPZ-fed Tnf ${ }^{-}$mice. As expected, OPC numbers were not increased in CPZ5 lesions of XPro1595-treated compared with vehicle-treated $\mathrm{Tnf}^{\prime-}$ mice, as they were in WT mice (Figure 4E).

To evaluate whether the increased OPC response in XPro1595-treated mice at CPZ5 was due to differences in OPC differentiation, we tested the effects of XPro1595 and solTNF on OPC differentiation in vitro, using mixed cortical neuron-glial cultures, which contain significant numbers (around 6\%) of 

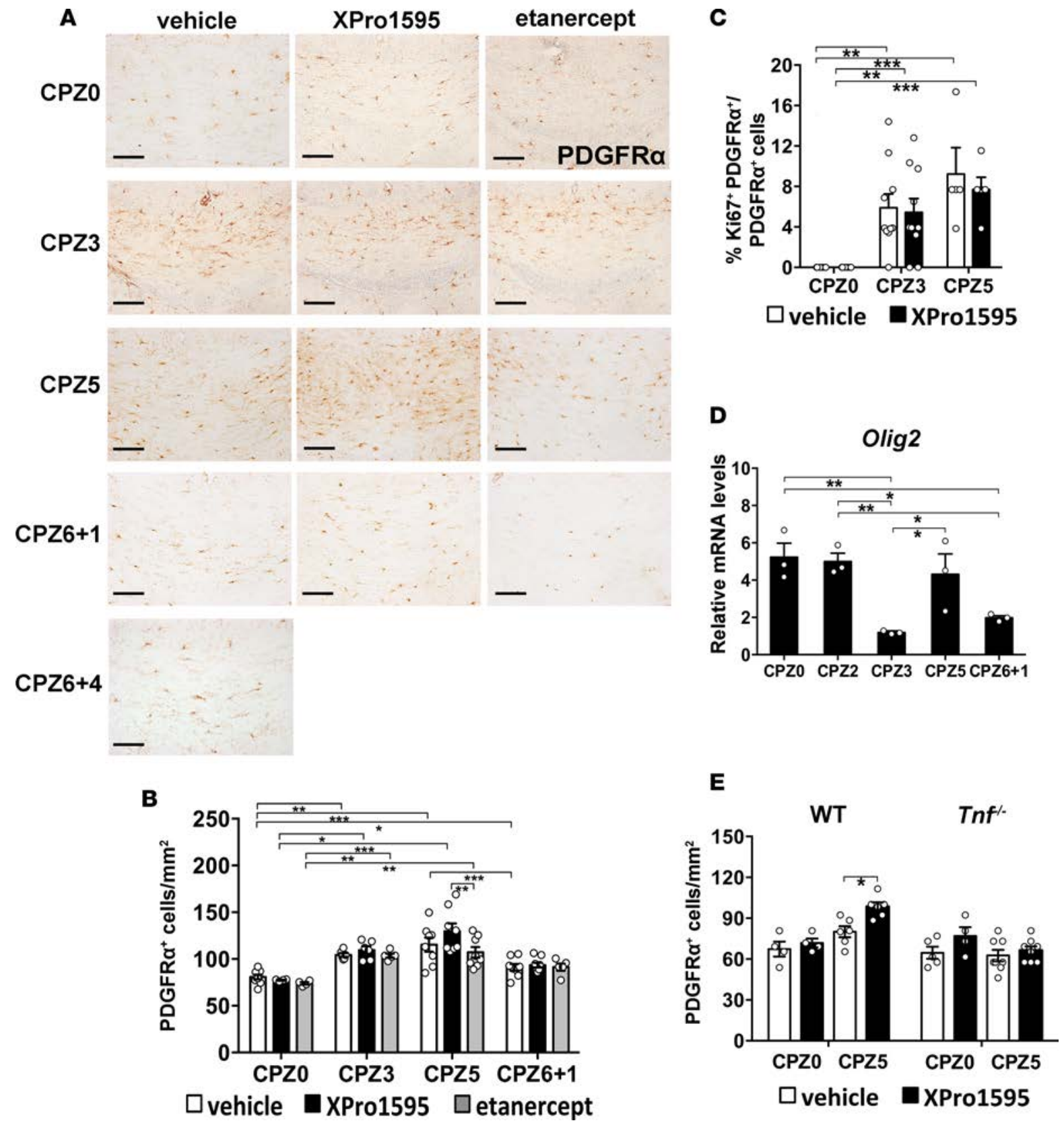

Figure 4. Inhibition of soluble TNF in brain increases OPC response in demyelinated lesions. (A) Platelet-derived growth factor receptor $\alpha$ (PDCFR $\alpha$ ) immunostaining of oligodendrocyte progenitor cells (OPC) in coronal paraffin sections of corpus callosum from representative vehicle- (left column), XPro1595(middle column), and etanercept-treated (right column) CPZO, CPZ3, CPZ5, CPZ6+1, and CPZ6+4 mice ( $n=8$ for vehicle-treated CPZO, $n=4$ for XPro1595treated CPZO, and $n=6-12$ for other time points). Scale bars: $100 \mu \mathrm{M}$. (B) Quantification of PDCFR $\alpha$-immunoreactive cells $/ \mathrm{mm}^{2}$ in the callosum in groups of mice represented in A. (C) Proportions of PDGFR $\alpha$-immunoreactive cells double-stained for the proliferation marker Ki67 in the callosum, in groups of mice represented in A. (D) Levels of Olig2 mRNA transcripts relative to Gusb in whole brain samples of vehicle-treated mice isolated from CPZO, CPZ2, CPZ3, CPZ5, and CPZ6+1 mice by quantitative PCR, ( $n=4$ per group). (E) Numbers of PDCFR $\alpha$-immunoreactive cells $/ \mathrm{mm}^{2}$ in the callosum of vehicle- and XPro1595treated CPZO and CPZ5 $\mathrm{Tnf}^{-/-}$and WT mice ( $n=6-8$ per group). The results shown are from one representative ([E] $\left.\operatorname{Tnf}^{-/}\right)$of two (D) or three ([A, B, C, and E] WT) independent experiments. Statistical significance after comparisons between numbers of PDCFR $\alpha$-immunoreactive cells in the different mouse strains ( $B$ and $\mathbf{E}$ ) and proportions of double positive cells (C) by two-way ANOVA with Bonferroni's test and mRNA levels (D) by ordinary one-way ANOVA with Tukey's test are shown. ${ }^{*} P<0.05,{ }^{*} P<0.01,{ }^{* *} P<0.001$. (B, C, D, and E) Circles show values for individual mice.

OPC immunostained for NG2 chondroitin sulfate proteoglycan (NG2) (Supplemental Figure 5, B and C). Cultures were incubated in the presence of XPro1595, murine solTNF, or medium alone, and the proportions of cells positive for NG2 (an OPC marker) and O4 (a marker of pre-myelinating and myelinating OLG), compared with total DAPI-stained cell nuclei, were measured. A small number of cells showed double immunoreactivity for NG2 and O4 under all culture conditions, suggestive of low-level OPC 
A
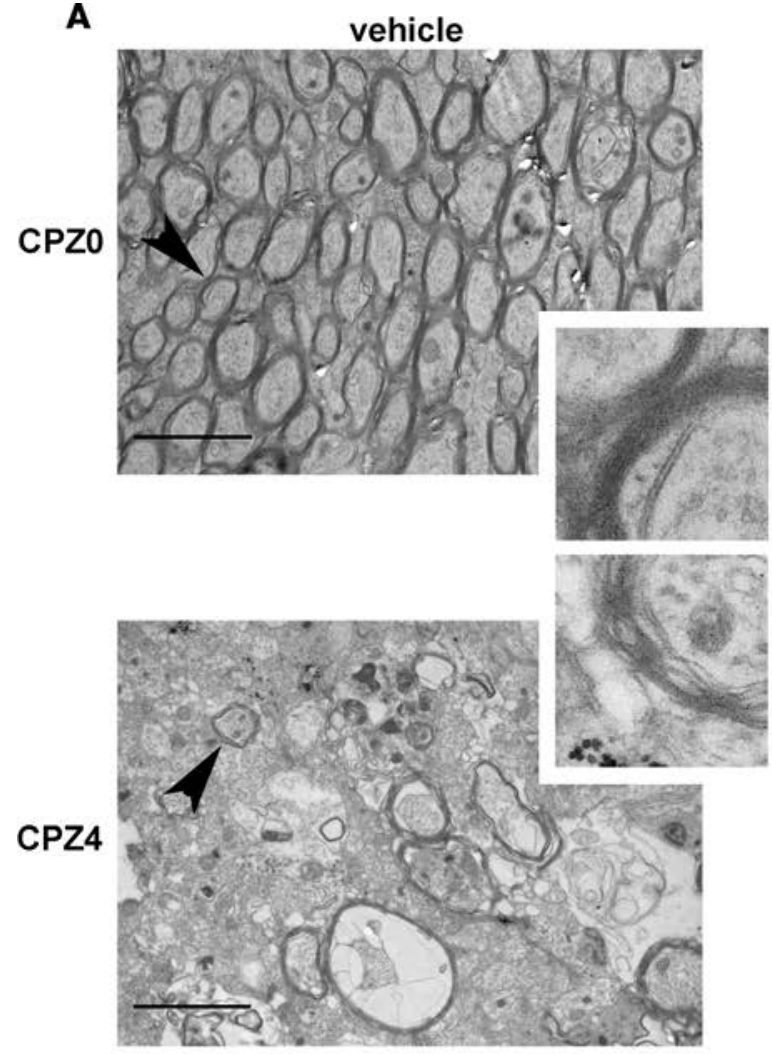

B

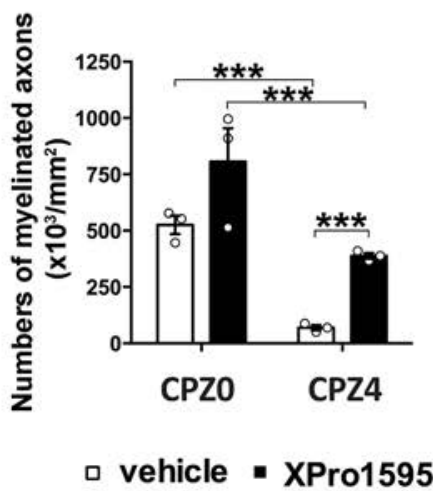

C

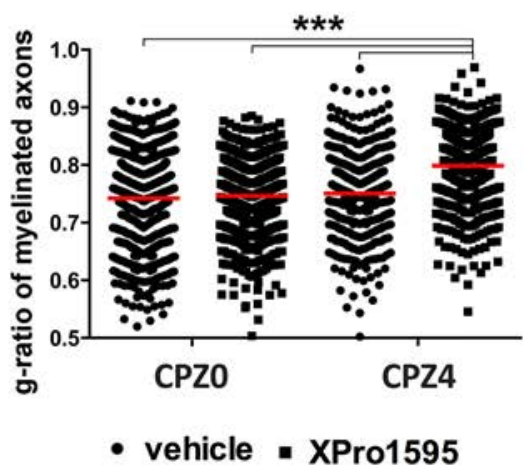

XPro1595
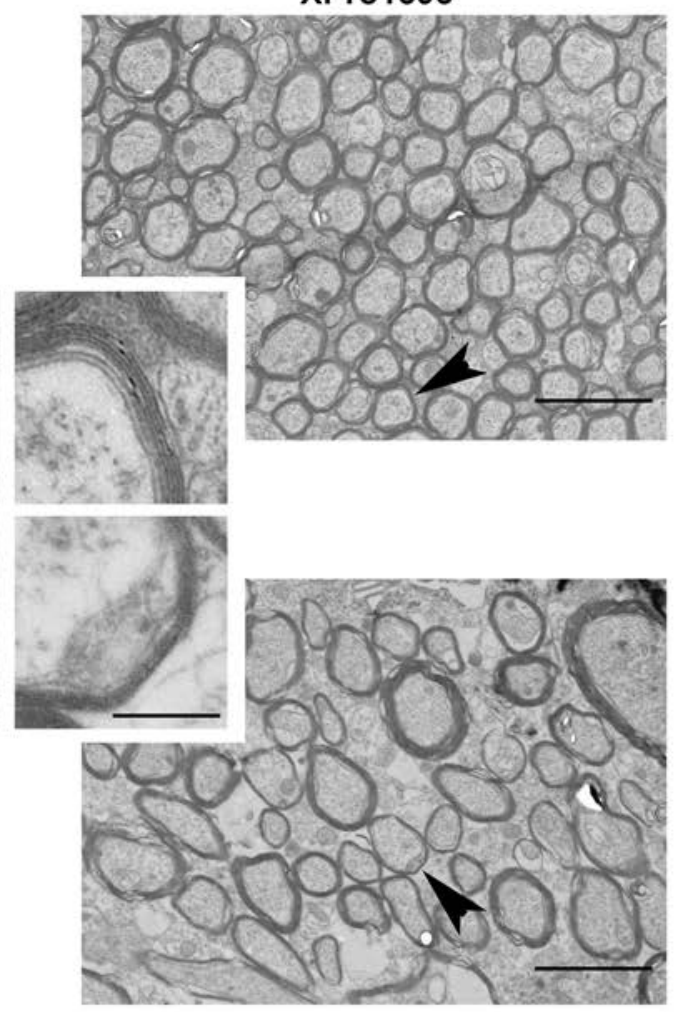

D

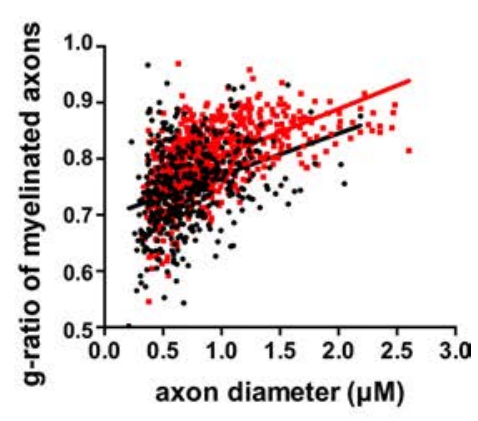

- CPZ4+vehicle

- CPZ4+XPro1595

Figure 5. Inhibition of soluble TNF in brain improves myelin compaction and promotes remyelination after CPZ-induced demyelination. (A) Electron micrographs of corpus callosum from vehicle- (left column) and XPro1595-treated (right column) CPZO and CPZ4 mice showing myelinated axons (arrowheads) ( $n=3$ per group). Scale bars: $1 \mu \mathrm{M}$. Insets show myelin at higher magnification. Scale bar: $150 \mathrm{nM}$. (B) Numbers of myelinated axons in corpus callosum $\left(1 \times 10^{3} / \mathrm{mm}^{2}\right)$. (C) Quantification of the g-ratios (axon area/fiber area) of myelinated fibers in the same mice as in $\mathbf{A}$. (D) Scatter plot showing distribution of g-ratios against axonal caliber in same mice as in $\mathbf{A}$. Statistical significance after comparisons between measurements of g-ratios (B) and numbers of myelinated axons (C) by one-way ANOVA with Tukey's test is shown. ${ }^{* *} P<0.001$. (B) Circles show values for individual mice.

differentiation in these conditions (Supplemental Figure 5B). The proportions of NG2-positive OPC and O4-positive OLG were very low, but equal in untreated, XPro1595, and TNF cultures at all time points measured.

These results indicate that solTNF does not alter the proliferation or differentiation capacity of OPC, but it inhibits their accumulation in the demyelinated lesions. They also suggest that TNF, by deduction tmTNF, is necessary for OPC accumulation in the demyelinating lesions.

Inhibition of solTNF in brain promotes remyelination and prevents decline of motor performance. To investigate whether the increased numbers of PDGFR $\alpha$-immunoreactive OPC (Figure 4, B and E) and myelin (Figure 1B) 




Figure 6. Inhibition of soluble TNF in brain prevents decline of motor performance. Rotarod test in vehicle- and XPro1595-treated naive and cuprizone-treated (CPZ-treated) mice at different time points. Vehicleand XPro1595-treated mice at experimental week 3 (naive vehicletreated, $n=9$; naive XPro1595-treated, $n=9$; vehicle-treated CPZ3, $n=14$ XPro1595-treated CPZ3, $n=14)$, week 4 ( $n=9, n=6, n=9, n=9$, respectively), weeks 5 and 6 ( $n=7, n=4, n=5, n=5$, respectively). The lengths of time the mice stayed on the accelerating cylinder ( $4-40 \mathrm{rpm}$ ) were recorded. Statistical significance after comparisons between all groups at each time point were performed using two-way ANOVA with Bonferroni's test. ${ }^{*} P<0.05$. Circles show values for individual mice.

in XPro1595-treated CPZ5 mice correlated with improved remyelination, we used electron microscopy to measure the numbers and g-ratios of myelinated axons and to evaluate the morphology of myelin in the corpus callosum at CPZ4. XPro1595-treated naive (CPZ0) mice showed normal myelin thickness and numbers of myelinated axons (Figure 5, A-C). CPZ feeding induced profound demyelination in vehicle-treated mice by CPZ4, as seen by the almost complete absence of myelinated axons (Figure 5, A and B), although the few remaining myelinated axons showed normal g-ratio, indicating they had not undergone demyelination (Figure 5C). XPro1595 treatment did not prevent demyelination, as seen by LFB staining at CPZ3 (Figure 1, $\mathrm{A}$ and $\mathrm{B}$ ) and loss of myelinated axons compared with XPro1595-treated naive mice by electron microscopy at CPZ4 (Figure 5B), but it significantly increased the numbers of thinly myelinated axons characteristic of remyelination (Figure 5A), as measured by higher g-ratios (Figure 5, C and D), and increased numbers of myelinated axons (Figure 5B) compared with vehicle controls. Regression analysis of g-ratios and axon diameters revealed that XPro1595 treatment enhanced remyelination of all axon sizes, including large-caliber axons, compared with vehicle treatment where small-caliber axons were preferentially remyelinated (Figure 5D). These results show that local inhibition of solTNF in the brain resulted in accelerated remyelination of demyelinated axons in the corpus callosum at CPZ4.

To test whether improved remyelination in XPro1595-treated mice was accompanied by better motor performance, we tested mice for overall balance, motor coordination, and motor learning using a rotarod apparatus following procedures described elsewhere (40). Motor function was reduced in vehicle-treated mice at CPZ5, as measured by marked reduction in time spent on the rotating cylinder, compared with naive mice (Figure 6). XPro1595 treatment did not affect the motor performance of naive CPZ0 mice at any time point tested and completely prevented the decline in motor function induced by CPZ, as measured by equal times spent on the rotating cylinder by XPro1595-treated CPZ mice and naive mice at all time points tested (Figure 6).

These results show that XPro1595 treatment promotes the remyelination of demyelinated axons in the callosum during CPZ feeding and prevents the CPZ-induced decline in motor function.

solTNF inhibition enhances myelin uptake by Mac3-immunoreactive phagocytes. In the acute CPZ model used here, OLG apoptosis starts as early as a few days after the initiation of CPZ and results in massive OLG death by week 4 (41) (Figure 7B). To determine whether solTNF contributes to OLG death, we treated B6 mice with twice-weekly s.c. injections of XPro1595 $(10 \mathrm{mg} / \mathrm{kg})$ or saline vehicle using a prophylactic protocol starting 2 weeks before CPZ feeding (6 injections), and we sacrificed mice at different time points for histopathological analysis. Overall, as with the therapeutic protocol (Figure 1), there was no significant difference in demyelination and OLG pathology in the corpus callosum between XPro1595- and vehicletreated mice. Thus, XPro1595 did not alter the progressive loss of CNPase-immunoreactive OLG (Figure 7, A and B), or the numbers of CNPase-immunoreactive OLG with apoptotic nuclei (Figure 7B), compared with vehicle-treated mice over time. Further, XPro1595 did not alter the presence of damaged myelin, which was measured by massively increased immunoreactivity for proteolipid protein (PLP) due to the unmasking of protein epitopes, compared with vehicle over time (Figure 7A. However, while XPro1595and vehicle-treated mice showed similar total numbers of Mac3-positive macrophages in the lesions, which progressively increased over time (Figure 7C), there were higher proportions of large macrophages containing PLP-immunoreactive myelin degradation products in XPro1595-treated lesions compared with vehicle controls (Figure 7, A and D). Further, XPro1595-treated mice at CPZ4 revealed areas highly infiltrated by 
A

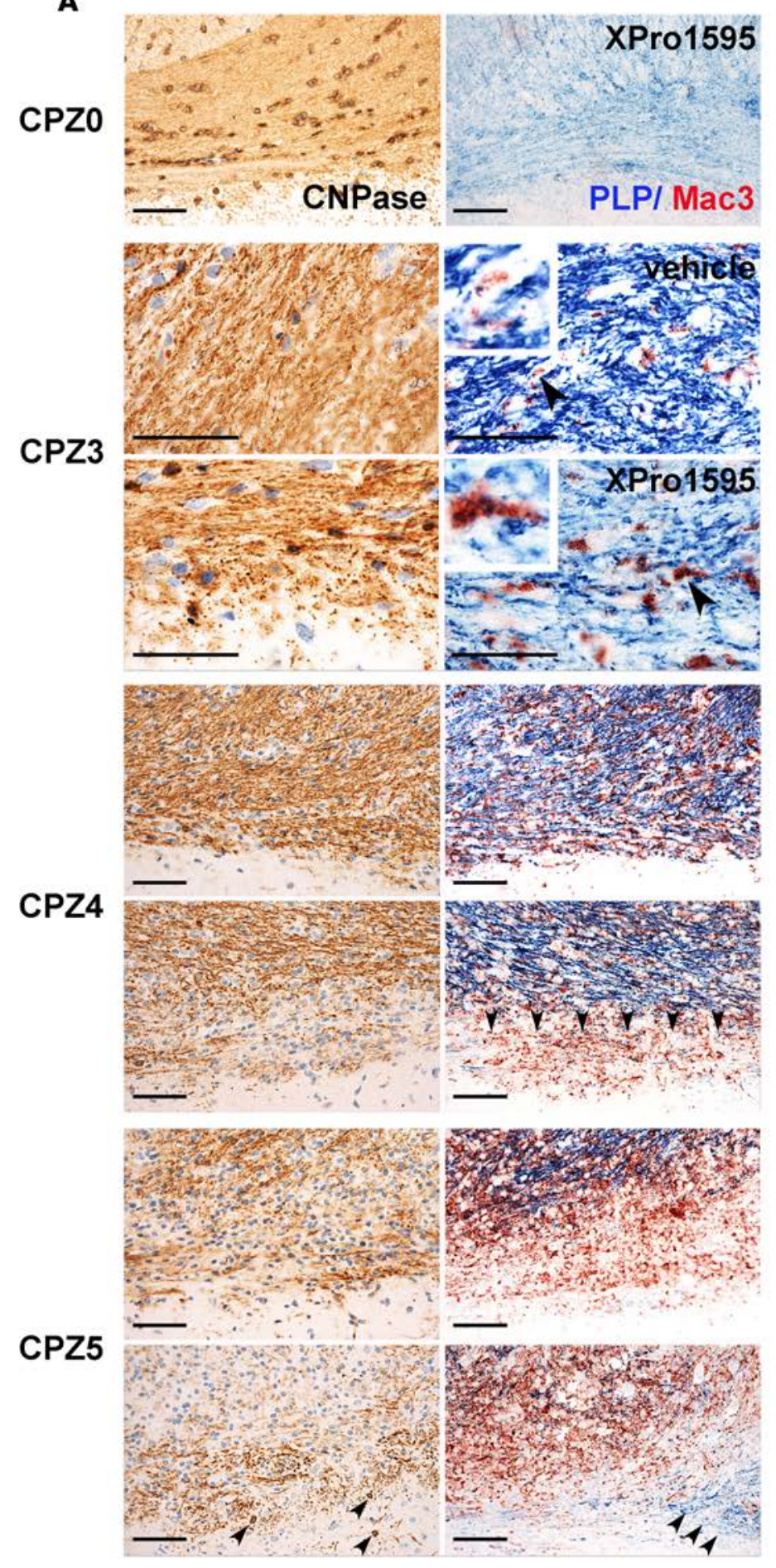

B

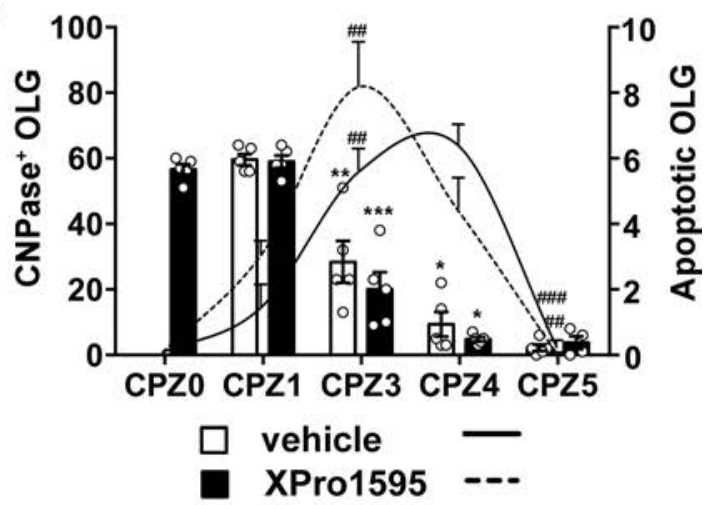

C

D

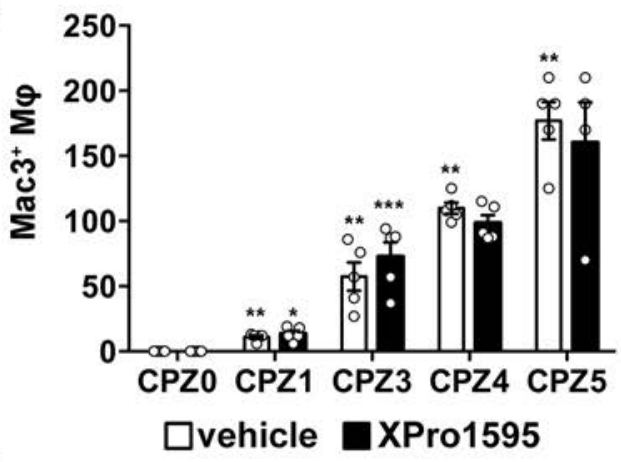

E

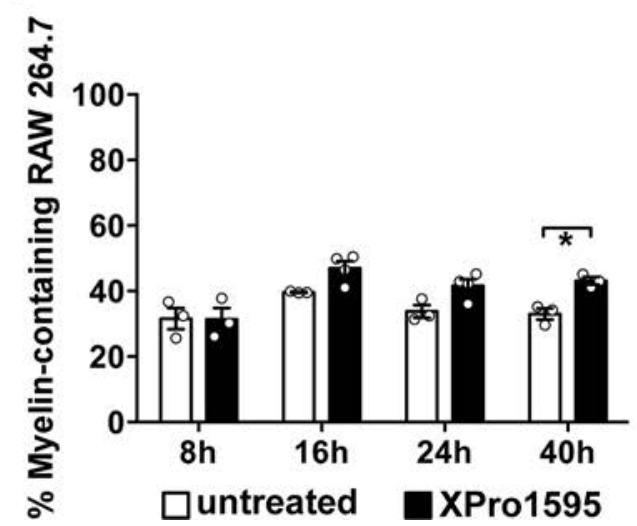

Figure 7. Inhibition of soluble TNF increases the clearance of myelin debris by phagocytic CNS macrophages and the production of new myelin in demyelinated lesions. (A) 2', 3'-cyclic nucleotide 3'-phosphodiesterase (CNPase) immunostaining of oligodendrocytes (OLG) (left column), proteolipid protein (PLP) immunostaining of myelin (right column, blue), and Mac3 immunostaining of CNS macrophages (right column, red) in coronal paraffin sections of corpus callosum from CPZO or CPZ-fed mice. At each time point, representative sections from vehicle-treated (CPZ3, CPZ4, CPZ5; upper rows) or XPro1595-treated (CPZO, CPZ3, CPZ4, CPZ5; lower rows) mice are shown ( $n=5$ per group). PLP immunoreactivity is massively increased in degenerating myelin that has lost OLG support (CPZ3, CPZ4, CPZ5). Scale bars: $100 \mu \mathrm{M}$. (B) Total numbers of CNPase-immunoreactive OLG/field ( $\times 40)($ bars, left axis), 
and CNPase-immunoreactive OLG with apoptotic nuclei/field, as detected by hematoxylin counterstaining of nuclei ( $\times 40)$ (curves, right axis). (C and D) Total numbers of Mac3-immunoreactive macrophages $(\mathrm{M} \varphi)$ /field $(\times 40)(\mathbf{C})$, and proportions of Mac3-immunoreactive macrophages containing PLPimmunoreactive myelin/field ( $\times 40)(\mathbf{D})$, in the callosum of the same mice represented in $\mathbf{A}$. (E) Proportions of mouse macrophage cells containing myelin basic protein-immunoreactive (MBP-immunoreactive) myelin assayed in the absence or presence of XPro1595. Statistical significance after comparisons between groups by two-way ANOVA with Bonferroni's test (B, C, and $\mathbf{D})$, or pairwise comparisons at each time point by Student's $t$ test (E), is shown. ${ }^{*} P<$ $0.05,{ }^{* *} P<0.01,{ }^{* * *} P<0.001,{ }^{\# \#} P<0.01,{ }^{\# \#} P<0.001$. (B, D, and $\mathbf{F}$ ) Asterisks denote statistical differences of each treatment group represented by bars with the same treatment group at the previous time point. Hash tags denote statistical differences of each treatment group represented on curves with the same treatment group at the previous time point. (A) Arrowheads show macrophages enlarged in insets (CPZ3), areas cleared of myelin debris (CPZ4), and new myelin (CPZ5).Circles show values for individual mice (B-E).

Mac3-positive macrophages that largely lacked CNPase and PLP-reactive myelin (Figure 7A). These were not observed in vehicle-treated animals, suggesting efficient clearance of myelin debris in XPro1595-treated animals. By CPZ5, both XPro1595- and vehicle-treated mice showed areas containing thin CNPase-immunostained myelin sheaths and weak expression of PLP, consistent with new myelin formation, and these remyelinating areas appeared larger in XPro1595-treated mice (Figure 7A). These results strongly indicate that selective inhibition of solTNF in the brain promotes myelin debris clearance by Mac3-immunoreactive macrophages (microglia), which in turn allows more-efficient remyelination of demyelinated axons.

To test whether solTNF directly affects macrophage phagocytic activity, we performed in vitro myelin phagocytosis assays using mouse macrophage cells in the absence and presence of XPro1595, and we measured myelin uptake by immunocytochemistry using anti-MBP antibodies (Supplemental Figure 6). Significantly larger proportions of macrophages containing MBP immunoreactivity were measured with XPro1595-supplemented medium compared with nonsupplemented medium by 40 hours of culture (Figure 7E). These data show that XPro1595 directly increases the ability of mouse macrophages to phagocytose myelin in vitro - and in vivo in CPZ-induced demyelinated lesions - thereby promoting remyelination of demyelinated axons.

\section{Discussion}

Here, we present evidence that local CNS production of solTNF inhibits the onset of remyelination and CNS repair in a CPZ model for MS by reducing the clearance of myelin debris by phagocytic CNS macrophages. Therapeutic administration of the selective solTNF inhibitor XPro1595 — which enters the intact CNS from the periphery in pharmacologically relevant concentrations - in CPZ-fed mice at the onset of histopathological changes did not halt the onset of demyelination, axon damage, or gliosis triggered by the toxin, but promoted the remyelination of demyelinated axons, resolved microgliosis, and halted progression of axon damage, thereby completely preventing the decline in motor function that is associated with CPZ feeding. The beneficial effects of solTNF blockade were associated with increased numbers of OPC in demyelinating lesions and profound early remyelination of demyelinated axons as a result of improved clearance of myelin debris by phagocytes in the presence of XPro1595. These results identify a deleterious role for local brain solTNF in inhibiting myelin regeneration. They also show a beneficial role for tmTNF in remyelination, with the therapeutic effects of XPro1595 being abolished in $\mathrm{Tnf}^{/-}$mice and replicated in $\operatorname{tm} \operatorname{Tnf}^{/ / \Delta}$ mice - findings consistent with previous reports that TNFR2, which efficiently transmits signals from tmTNF (32), is necessary for OLG proliferation and remyelination in this model (16).

It was important to exclude the possibility that XPro1595 treatment ameliorated demyelination in response to CPZ feeding. First, histochemical and immunochemical analyses showed that demyelination developed equally in the corpus callosum of mice treated therapeutically or prophylactically with XPro1595 and vehicle. Second, the numbers of apoptotic OLG were equal in the corpus callosum of mice treated prophylactically with XPro1595 or vehicle at all time points of CPZ administration, showing that solTNF does not contribute to OLG death in this model. Third, early demyelination developed similarly in $T_{n f^{-1}}$ (CPZ5) and tmTNF ${ }^{4 / 4}$ (CPZ3) mice as in vehicle-treated WT mice. In the CPZ model, OLG death occurs in two waves. A first early wave starts days after the initiation of CPZ feeding. This is thought to result from combined metabolic stress (42) and local NO production (43), which directly sensitize OLG to apoptosis, as demonstrated by the sharply reduced expression of myelin genes $(26,27)$ (Figure 4D and Supplemental Figure 1B), and the presence of activated caspase 3 in OLG (41). Subsequently, accumulating inflammatory cells, predominantly activated microglia (36) but also infiltrating immune cells such as CXCR2-positive neutrophils (24), IL-17 signaling in CNS resident cells (44), and immune mediators such as chemokine (C-C motif) ligand 3/macrophage inflammatory protein-1 $\alpha(\mathrm{Ccl} 3)(45)$, contribute to massive 
OLG apoptosis by 4 weeks of dietary CPZ (Figure 7B) and lead to demyelination in the corpus callosum. The specific molecular triggers of OLG apoptosis in this model, however, remain to be identified, and as shown here, do not include TNF.

TNF and TNFR2 both promote CNS remyelination and repair in the EAE and CPZ models of CNS demyelination. In the CPZ model, TNF and TNFR2 expression are upregulated in the brain during the demyelination process, and although the absence of TNF in mice does not prevent demyelination or microglia proliferation, it significantly delays remyelination in the corpus callosum that normally takes place within weeks after withdrawal of CPZ (16). This report further shows that TNF and TNFR2 are both necessary for the efficient proliferation of NG2-immunoreactive OPC and remyelination of demyelinated axons in this model (16). Our finding here that the local production of solTNF in the CNS during CPZ demyelination inhibits remyelination, therefore, at first appears paradoxical. However, the observation that solTNF inhibition in WT mice allowed profound remyelination at a very early time point (CPZ4), while CPZ was still in the diet, and that this effect was abolished in $\mathrm{Tnf}^{1-}$ mice, provides strong evidence that solTNF inhibits tmTNF-dependent remyelination and is therefore fully consistent with previous findings. Similarly, XPro1595 administration protects mice against EAE disease symptoms. This is associated with preservation of myelin morphology, increased numbers of NG2-positive OPC and remyelinating axons, and neuroprotection $(19,46)$. tmTNF signals efficiently through TNFR2, and the recent finding that a human TNFR2 transgene is predominantly expressed on OPC rather than on differentiated OLG in primary OLG cultures isolated from mice - and that activation of this receptor protects OPC against oxidative stress (47) - provides evidence that tmTNF might signal directly through TNFR2 on OLG lineage cells to mediate remyelination. Alternatively, tmTNF might signal indirectly through TNFR2 expressed on CNS macrophages in mice and patients with MS (19) to mediate remyelination. A requirement for OLG-specific TNFR2 expression for mediating remyelination following CNS demyelination was recently demonstrated in vivo in the EAE model (20). Taken together, the available data support that solTNF and TNFR1 inhibit remyelination, that tmTNF and TNFR2 promote OPC proliferation and remyelination, and that selective inhibition of solTNF (and TNFR1) unmasks the full remyelinating potential of tmTNF (and TNFR2).

The cellular mechanism underlying the inhibitory effect of solTNF on remyelination does not appear to involve alteration of OLG apoptosis or OPC proliferation, differentiation, or recruitment in response to CPZ-induced demyelination. While OPC responses to demyelination were increased in XPro1595-treated mice, this could not be explained by an increase in OPC proliferation in vivo, or of OPC differentiation in vitro. On the other hand, our in vivo and in vitro data clearly show a role for solTNF in inhibiting the beneficial activity of phagocytic CNS macrophages (microglia) in clearing myelin debris. solTNF is classically associated with chronically activated proinflammatory microglia and tissue damage in the CNS (17). Accordingly, XPro1595 treatment ameliorates microgliosis and primary pathology in a range of experimental models, including EAE (19, 46), cerebral ischemia (48), and Parkinson's disease (49), and here resolved microgliosis in demyelinated lesions - findings that are all consistent with a role for solTNF in driving CNS inflammation. Interestingly, XPro1595-treated naive mice showed increased brain expression of Chil3, which encodes a protein associated with host defense (37) and is used as a marker for beneficial alternatively activated macrophages. Previous work also shows that XPro1595 treatment induces a transient activation of immune genes in the spinal cord at the onset of EAE, in parallel to having protective effects on OLG and neuronal gene expression (50). Here, although XPro1595 treatment did not alter the numbers of Mac3-positive macrophages in early demyelinating lesions, it increased the numbers and size of macrophages containing myelin degradation products and facilitated the clearance of myelin debris and remyelination in corpus callosum lesions. It also enhanced myelin phagocytosis by macrophages in vitro. Macrophages are known to be important for the remyelination response to demyelination. They are necessary for early stages of remyelination and for the recruitment of PDGFR $\alpha$-immunoreactive OPC in a lysolecithin-induced demyelination model (refs. 12, 51). Macrophages are the main cell type responsible for phagocytotic clearance of myelin debris $(52,53)$, which contains inhibitors of remyelination and, in turn, inhibits OPC differentiation in vitro (54) and in vivo (13). Studies in MS lesions provided early evidence that different macrophages might be associated with different types of MS lesions $(55,56)$. Thus, macrophages in active demyelinating lesions mainly express acute-stage inflammation markers and contain myelin-immunoreactive debris, while macrophages in remyelinating lesions are lipid-laden, often PAS-positive foamy cells that contain myelin breakdown products $(55,56)$, similar to those seen in the XPro1595-treated lesions. Although we were not able to distinguish between microglia and blood-derived 
macrophages in this study, since both cell types can give rise to macrophages in the CNS, the finding that etanercept - which inhibits solTNF and tmTNF in the periphery - did not alter demyelination or remyelination strongly suggests that XPro1595 exerts its beneficial effects via CNS microglia. In support of this, recent studies in a model of spinal cord injury show that TNF plays a critical role in maintaining the proinflammatory phenotype of microglia/macrophages and inhibits a switch toward an antiinflammatory prorepair phenotype in the spinal cord following injury $(57,58)$.

Our data provide evidence that local production of solTNF in the brain in a mouse model for demyelination and remyelination not only promotes the proinflammatory function of microglia during demyelination, but also inhibits the beneficial function of phagocytic CNS macrophages (microglia) in clearing myelin debris and permitting remyelination, a process mediated at least in part by tmTNF. Our results also suggest that selective inhibition of solTNF by CNS-penetrating molecules such as XPro1595 represents a promising therapeutic approach for promoting remyelination and neuroprotection in diseases such as MS.

\section{Methods}

Mice. B6 mice were used for CPZ-induced demyelination. Tnf ${ }^{\prime-}$ mice (59) were backcrossed for at least 12 generations onto the $\mathrm{B} 6$ background. $\operatorname{tm} \operatorname{Tnf}^{/ / 4}$ mice (donated by Bernard Ryffel, University of Orleans and CNRS, INEM, Molecular Immunology, Orleans, France) were generated directly onto the B6 background (33). Non-transgenic littermates were used as controls in all experiments. Mice were maintained under specific pathogen-free conditions in the experimental animal unit of the Hellenic Pasteur Institute.

CPZ model of demyelination and remyelination. Eight- to 10 -week-old male WT, Tnf ${ }^{\prime-}$, or $\mathrm{tm} \operatorname{Tnf}^{1 / 4}$ mice were fed ad libitum with $0.2 \% \mathrm{w} / \mathrm{w}$ CPZ (Sigma-Aldrich; catalog C9012) in powdered standard mouse chow as described (21). Consistent with this model, we measured hallmark clinical features of disease in the corpus callosum of CPZ-treated WT mice at the end of CPZ3 (microgliosis, astrocytosis, OPC proliferation, and demyelination), CPZ5 (maximal demyelination, decline in motor performance), CPZ6+1 (measurable remyelination), and $\mathrm{CPZ6}+4$ (almost-complete remyelination).

Treatments with TNF inhibitors. CPZ-fed and naive control mice were treated with twice-weekly s.c. injections of XPro1595 (10 mg/kg; Xencor) (18), etanercept (10 mg/kg; Amgen) (30), or saline vehicle. Experiments corresponding to Figures 1-6 were performed in mice treated using a therapeutic protocol, in which injections started at the end of CPZ2, at the onset of demyelination (21). In one set of experiments (Figure 7), to test the effect of TNF inhibitors on OLG apoptosis at different time points of CPZ feeding, mice were treated prophylactically, with injections starting 2.5 weeks before CPZ feeding.

Rotarod test. Mice were tested for overall balance, motor coordination, and motor learning using a rotarod apparatus (ENV-575M, Med Associates Inc.), as described (40). Briefly, mice were placed on the rotating cylinder at an accelerating speed of 4-40 rpm and trained for 3 sessions per day for 3 consecutive days (days 11, 12, and 13 of CPZ feeding), prior to the first test on day 14 (CPZ2), and tested once weekly from then on. The times mice remained on the cylinder were recorded.

Histology and IHC. Mice were transcardially perfused with ice-cold 4\% paraformaldehyde in PBS under deep anaesthesia. Brains were removed, post-fixed in the same fixative overnight at $4^{\circ} \mathrm{C}$, embedded in paraffin or equilibrated in 15\% sucrose, and snap frozen for cryostat sectioning. For histology and IHC, serial coronal paraffin sections $(3-5 \mu \mathrm{M})$ were cut through the corpus callosum corresponding to Sidman sections 295-305, and comparative analyses were made in the midline corpus callosum (area annotated in Figure 2A, vehicle-treated CPZ0 sample). Sections were stained with LFB for demyelination, and Biel silver impregnation for axonal damage according to standard procedures (60). IHC was performed as described (60) using the primary antibodies mouse anti-2', 3'-cyclic nucleotide 3'-phosphodiesterase (CNPase) mAb (1/500; clone SMI-91; Covance), mouse SMI 32 mAb (1/1,000; clone SMI-32; Covance), rabbit anti-ionized calcium-binding adaptor molecule 1 (Iba1) (1/500; Wako chemicals; catalog 019-19741), rabbit antiGFAP (1/300; Dako; catalog Z0334), rabbit anti-platelet-derived growth factor receptor alpha (PDGFR $\alpha$ ) (1/150; donated by W. Stallcup, Sanford Burnham Prebys Institute, La Jolla, California, USA); followed by biotinylated secondary Abs, horseradish peroxidase-labeled avidin-biotin complex, and visualized using DAB (all from Vector Laboratories). Double immunostaining for proteolipid protein (PLP) and Mac3 was performed based on previous methods (60) using mouse anti-PLP $(1 / 1,000$; clone plpc1; Serotec), detection by alkaline phosphatase-conjugated anti-mouse secondary Ab (1/200; Jackson ImmunoResearch; catalog 115-056-068), and visualization by fast blue (blue reaction product), followed by antigen retrieval in $10 \mathrm{mM}$ EDTA buffer ( $\mathrm{pH} 8.5$ ) for 1 hour at $95^{\circ} \mathrm{C}-100^{\circ} \mathrm{C}$ in a household food steamer, incubation with 
rat anti-Mac3 mAb (1/200; clone M3/84; Serotec), and detection by biotinylated anti-rat Ab (1:500; Jackson ImmunoResearch; catalog 112-005-003), followed by avidin-peroxidase and visualization by 3-amino9-ethylcarbazole (red reaction product). Double immunofluorescence staining for proliferating OPC was performed using a mixture of rabbit anti-PDGFR $\alpha$ (as above) and mouse anti-Ki67 mAb (1/50; clone B56; BD Biosciences), followed by a mixture of AlexaFluor 568 anti-rabbit IgG (1/500; Molecular Probes; cata$\log$ A11011) and AlexaFluor 488 anti-mouse IgG (1/500; Molecular Probes; catalog A11001) secondary antibodies. Nuclei were counterstained with DAPI (1/1,000; Invitrogen; catalog D1306).

Histology quantification. Tissue sections were viewed with an Olympus BX-50 microscope and images captured with an Olympus DP71 microscope digital camera using cell^A imaging software (Soft Imaging System $\mathrm{GmbH}$ ). Quantitative histopathological analysis was performed using Image J software. Demyelination was measured as loss of LFB staining in coronal sections of whole corpus callosum using a semiquantitative method, as described (16), and loss of CNPase immunostaining in the midline corpus callosum by densitometry (area was used for all midline measurements shown in Figure 2A, vehicle-treated CPZ0 mouse). Axonal damage was measured as area covered by SMI 32 immunostaining in the midline corpus callosum. Microglia and OPC responses were measured as the areas covered by Iba1 and PDGFRo immunoreactivity, respectively, and as the numbers of positive cells by stereology (Iba1-immunoreactive cells $/ \mathrm{mm}^{3}$ ) or cell counting (PDGFR $\alpha$-immunoreactive cells $/ \mathrm{mm}^{2}$ ) in the midline corpus callosum. Corpus callosum volumes in treatment groups were measured as described (61) using $30 \mu \mathrm{M}$ serial coronal Cresyl Violet-stained cryostat sections taken between Bregma -1.3 and -2.3 with cell^ $\mathrm{A}$ imaging software.

Stereology. Iba1-immunoreactive microglia/macrophage density in the corpus callosum was estimated by an unbiased stereological quantification method utilizing the optical fractionator principle incorporated in Stereo Investigator v10.0 software (MicroBrightField Bioscience), using a light microscope (Leica, DMRA2) equipped with a motorized stage (MAC5000, Lud1 Electronic Products Ltd.) and top-mounted camera (QImaging), as described (62). Briefly, every fifth coronal free-floating cryostat section $(30 \mu \mathrm{M})$ taken through the corpus callosum between Bregma -1.3 and -2.3 was immunostained for Iba1 and counterstained with Cresyl Violet, and Iba1-immunoreactive microglia in the whole defined callosum volume were counted.

Electron microscopy. Vehicle- and XPro1595-treated naive (CPZ0) and CPZ4 mice were perfused with $4 \%$ paraformaldehyde $/ 2.5 \%$ glutaraldehyde and post-fixed in the same fixative, and $300 \mu \mathrm{M}$ thick sagittal vibratome slices were prepared using a Leica VT1200S vibratome (Leica). The region of interest was punched out, postfixed with $2 \% \mathrm{OsO}_{4}$ (Science Services), and embedded in Epon (Serva). Ultrathin sections $(50 \mathrm{~nm}$ ) were collected onto copper grids (Gilder Grids Ltd.), coated with formvar (Plano), and stained with $4 \%$ aqueous uranyl acetate (SPI-Chem) and lead citrate (63). Electron micrographs of cross-sectioned axons in the caudal corpus callosum were taken with a LEO EM912 omega electron microscope (Zeiss) using an on-axis 2k CCD camera (TRS). Fifteen random pictures (at 6,300×) were taken as multiple image assemblies from each mouse, and all fibers per picture were analyzed using Image J (NIH) software. Three mice per group per time point were analyzed. Data are expressed as g-ratios (axon area/myelinated axon area) for all myelinated axons measured in callosum of each mouse in each group $\left(1 \times 10^{3} / \mathrm{mm}^{2}\right)$.

Total RNA isolation and quantitative PCR. Total RNA was extracted from whole brains using TRIzol reagent (Thermo Fisher Scientific; catalog 15596026) per manufacturer's instructions. DNase-treated RNA samples were subjected to quantitative PCR (qPCR) using a QuantiFast SYBR Green RT-PCR kit (Qiagen; catalog 204154), per manufacturer's instructions. All reactions were performed using a LightCycler (Roche Diagnostics) as described (64). Values were normalized using the respective values for Gusb, or Gapdh (for Arg1, Chil3, Il1B, and Nos2). Results were analyzed using LightCycler software version 3.5 (Roche Diagnostics). QuantiTect Primer Assays were used for Arg1 (Qiagen primer code; Mm_Arg1_1_SG), Ccl2 (Mm_Ccl2_1_SG), Chil3 (Mm_Chil3_1_SG), Cxcl16 (Mm_Cxcl16_1_SG), Gapdh (Mm_Gapdh_3_SG), Gusb (Mm_Gusb_1_SG), Il1 $\left(\mathrm{mM} \_I 11 b \_2 \_S G\right), M b p$ (Mm_Mbp_1_SG), Nos2 (mM_LOC673161_1_SG), Olig2 (Mm_Olig2_1_SG), Snap25(Mm_Snap25_2_SG), and Tnf(Mm_Tnf_1_SG).

Measurement of human TNF in blood and tissues. Blood was collected by heart puncture from deeply anesthetized vehicle- and XPro1595-treated naive (CPZ0) and CPZ5 mice, and sera were collected and snap frozen. Mice were briefly perfused with ice-cold PBS, and brains were removed and snap frozen. Levels of human TNF (XPro1595) were measured in sera and brain lysates using a human TNF V-PLEX immunoassay (Meso Scale Discovery). Levels of human TNFR2 Fc fusion protein (etanercept) were measured in sera and brain lysates using a human TNFR2 V-PLEX immunoassay (Meso Scale Discovery). 
Mixed cortical neuron-glial cultures. Primary mixed cortical neuron-glial cultures containing OLG lineage cells were prepared from fetal B6 mice at embryonic day 14 (E14), based on established protocols (65). Briefly, dissociated cortical cells were grown on poly-D-lysine-coated (Sigma-Aldrich; catalog P6407) coverslips in 48-well plates in high-glucose DMEM (Sigma-Aldrich; catalog D5671) supplemented with 5\% horse serum (Thermo Fisher Scientific; catalog 16050122) and 5\% FBS (Thermo Fisher Scientific; catalog 10270106). After 7 days in vitro (DIV7), recombinant murine TNF (100 ng/ml) (R\&D systems; catalog 410-MT-010), or XPro1595 (100 ng/ml), was added for 24 or 48 hours. Cells were fixed with $2 \%$ paraformaldehyde in PBS and immunostained using a mixture of rabbit anti-NG2 (1/100; donated by W. Stallcup, Sanford Burnham Prebys Institute, La Jolla, California, USA) and mouse anti-O4 (1/25; clone 81; Merck Millipore) antibodies, followed by a mixture of AlexaFluor 568 anti-rabbit IgG (1/500; Molecular Probes; catalog A11011) and AlexaFluor 488 anti-mouse IgG (1/500; Molecular Probes; cata$\log$ A11001) secondary Abs. Nuclei were counterstained with DAPI (1/1,000; Invitrogen; catalog D1306). Cells were viewed with an Olympus Time-Lapse microscope ( $\times 40$ objective), and 20 images per well, in at least three separate cultures per condition, were captured using cell ${ }^{\wedge} \mathrm{R}$ imaging software (Soft Imaging System $\mathrm{GmbH}$ ). Total numbers of DAPI-stained nuclei, NG2-positive OPC, and O4-positive OLG per well were counted and expressed as proportions of DAPI-stained cell nuclei.

Myelin phagocytosis assay. Myelin was isolated from B6 mice as described (66). RAW 264.7 macrophage cells were grown according to supplier's instructions (ECACC; catalog ATL02001) and seeded at $4 \times 10^{5}$ cells/well together with myelin at $5 \mu 1 /$ well in 24 -well plates. Cells were incubated without or with XPro1595 (100 ng/ml) for different lengths of time (0-40 hours), washed, fixed with 2\% paraformaldehyde in PBS for 20 min, immunostained using rabbit anti-MBP antibody (1/200; Dako; catalog A0623) followed by AlexaFluor 568 anti-rabbit IgG (1/500; Molecular Probes; catalog A11011), and counterstained with DAPI.

Statistics. Data in figures are presented as mean \pm SEM and individual data points. All statistical analyses were performed using Prism 6 (GraphPad). Statistical analyses to compare the mean values for multiple groups were performed using ordinary one-way or two-way ANOVA combined with a post-test as stated in figure legends. Pairwise comparisons between individual groups was performed using a 2-tailed unpaired $t$ test. Values were accepted as significant at $P \leq 0.05$.

Study approval. All experimental protocols were evaluated and approved by the Institutional Protocol Evaluation Committee in Athens, Greece, and the national Hellenic authorities, and conformed to European Union guidelines. Licenses were issued by the Official Hellenic Veterinary Authorities.

\section{Author contributions}

MK was responsible for day-to-day management of the project, designed and performed all in vivo experiments and in vitro assays, and analyzed data shown in Figures 1-6 and Supplemental Figures 1, 2, 4, 5, and 6. WM performed TEM shown in Figure 5. CB and MGT performed the hTNF immunoassay and analyzed data shown in Figure 1D and Supplemental Figure 3. DES provided XPro1595 and etanercept. HL performed, analyzed, and interpreted the histological analyses of demyelinating lesions shown in Figure 7. LP conceived the project, designed the experiments, interpreted the results, and wrote the paper. All authors critically reviewed and edited the final manuscript.

\section{Acknowledgments}

We thank Ray J. Tesi (INmuneBio) and Klaus-Armin Nave for their support; William Stallcup for providing Abs to PDGFR $\alpha$ and NG2; Bernard Ryffel for providing tmTnf/s mice; Nikolaus Plesnila, Maria Xilouri, and Maria Nikatou for advice with sterology; and Bogusia Sadowski, Torben Ruhwedel, and Martin Meschkat for technical help with the TEM. We would also like to thank Florentia Papastefanaki and Eirini Fragkiadaki for help with the rotarod, and Irini Papazian for training in primary neuron cultures. This research was supported by the European Commission through NeuroSign Capacities Framework 7 project grant 264083, by the Hellenic General Secretariat of Research and Technology through the National Action Cooperation project grant 09SYN-21-609 and the Operational Program project grant KRIPIS MIS450598, and by the Hellenic Academy of Neuroimmunology (HELANI) through a fellowship to MK from Teva Pharmaceutical Industries LTD. Part of the TEM work (Wiebke Möbius, Bogusia Sadowski, and Martin Meschkat) was supported by the Cluster of Excellence and DFG Research Center Nanoscale Microscopy and Molecular Physiology of the Brain, Göttingen, Germany. 
Address correspondence to: Lesley Probert, Laboratory of Molecular Genetics, Hellenic Pasteur Institute, 11521 Athens, Greece. Phone: 30.210.6478866; E-mail: lesley.probert@gmail.com.

CB's present address is: Takeda Pharmaceuticals U.S.A. Inc., Deerfield, Illinois, USA.

1. Franklin RJ, Kotter MR. The biology of CNS remyelination: the key to therapeutic advances. J Neurol. 2008;255 Suppl 1:19-25

2. Farh KK, et al. Genetic and epigenetic fine mapping of causal autoimmune disease variants. Nature. 2015;518(7539):337-343.

3. International Multiple Sclerosis Genetics Consortium, et al. Genetic risk and a primary role for cell-mediated immune mechanisms in multiple sclerosis. Nature. 2011;476(7359):214-219.

4. Lopez-Diego RS, Weiner HL. Novel therapeutic strategies for multiple sclerosis--a multifaceted adversary. Nat Rev Drug Discov. 2008;7(11):909-925

5. Bramow S, et al. Demyelination versus remyelination in progressive multiple sclerosis. Brain. 2010;133(10):2983-2998

6. Patrikios P, et al. Remyelination is extensive in a subset of multiple sclerosis patients. Brain. 2006;129(Pt 12):3165-3172.

7. Mahad DH, Trapp BD, Lassmann H. Pathological mechanisms in progressive multiple sclerosis. Lancet Neurol. 2015;14(2):183-193.

8. Franklin RJ, Ffrench-Constant C. Remyelination in the CNS: from biology to therapy. Nat Rev Neurosci. 2008;9(11):839-855.

9. Foote AK, Blakemore WF. Inflammation stimulates remyelination in areas of chronic demyelination. Brain. 2005;128(Pt 3):528-539.

10. Huitinga I, van Rooijen N, de Groot CJ, Uitdehaag BM, Dijkstra CD. Suppression of experimental allergic encephalomyelitis in Lewis rats after elimination of macrophages. J Exp Med. 1990;172(4):1025-1033.

11. Yamasaki R, et al. Differential roles of microglia and monocytes in the inflamed central nervous system. J Exp Med. 2014;211(8):1533-1549.

12. Kotter MR, Setzu A, Sim FJ, Van Rooijen N, Franklin RJ. Macrophage depletion impairs oligodendrocyte remyelination following lysolecithin-induced demyelination. Glia. 2001;35(3):204-212.

13. Kotter MR, Li WW, Zhao C, Franklin RJ. Myelin impairs CNS remyelination by inhibiting oligodendrocyte precursor cell differentiation. J Neurosci. 2006;26(1):328-332.

14. Selmaj K, Raine CS. Tumor necrosis factor mediates myelin damage in organotypic cultures of nervous tissue. Ann N Y Acad Sci. 1988;540:568-570.

15. Akassoglou K, et al. Oligodendrocyte apoptosis and primary demyelination induced by local TNF/p55TNF receptor signaling in the central nervous system of transgenic mice: models for multiple sclerosis with primary oligodendrogliopathy. Am J Pathol. 1998;153(3):801-813.

16. Arnett HA, Mason J, Marino M, Suzuki K, Matsushima GK, Ting JP. TNF alpha promotes proliferation of oligodendrocyte progenitors and remyelination. Nat Neurosci. 2001;4(11):1116-1122.

17. Probert L. TNF and its receptors in the CNS: The essential, the desirable and the deleterious effects. Neuroscience. 2015;302:2-22.

18. Steed PM, et al. Inactivation of TNF signaling by rationally designed dominant-negative TNF variants. Science. 2003;301(5641):1895-1898.

19. Brambilla R, et al. Inhibition of soluble tumour necrosis factor is therapeutic in experimental autoimmune encephalomyelitis and promotes axon preservation and remyelination. Brain. 2011;134(Pt 9):2736-2754.

20. Madsen PM, et al. Oligodendroglial TNFR2 Mediates Membrane TNF-Dependent Repair in Experimental Autoimmune Encephalomyelitis by Promoting Oligodendrocyte Differentiation and Remyelination. J Neurosci. 2016;36(18):5128-5143.

21. Hiremath MM, Saito Y, Knapp GW, Ting JP, Suzuki K, Matsushima GK. Microglial/macrophage accumulation during cuprizone-induced demyelination in C57BL/6 mice. J Neuroimmunol. 1998;92(1-2):38-49.

22. Matsushima GK, Morell P. The neurotoxicant, cuprizone, as a model to study demyelination and remyelination in the central nervous system. Brain Pathol. 2001;11(1):107-116.

23. Bakker DA, Ludwin SK. Blood-brain barrier permeability during Cuprizone-induced demyelination. Implications for the pathogenesis of immune-mediated demyelinating diseases. J Neurol Sci. 1987;78(2):125-137.

24. Liu L, et al. CXCR2-positive neutrophils are essential for cuprizone-induced demyelination: relevance to multiple sclerosis. Nat Neurosci. 2010;13(3):319-326.

25. Lindner M, Fokuhl J, Linsmeier F, Trebst C, Stangel M. Chronic toxic demyelination in the central nervous system leads to axonal damage despite remyelination. Neurosci Lett. 2009;453(2):120-125.

26. Jurevics $\mathrm{H}$, et al. Alterations in metabolism and gene expression in brain regions during cuprizone-induced demyelination and remyelination. J Neurochem. 2002;82(1):126-136.

27. Morell P, et al. Gene expression in brain during cuprizone-induced demyelination and remyelination. Mol Cell Neurosci. 1998;12(4-5):220-227.

28. Zalevsky J, et al. Dominant-negative inhibitors of soluble TNF attenuate experimental arthritis without suppressing innate immunity to infection. J Immunol. 2007;179(3):1872-1883.

29. Mogi M, Harada M, Riederer P, Narabayashi H, Fujita K, Nagatsu T. Tumor necrosis factor-alpha (TNF-alpha) increases both in the brain and in the cerebrospinal fluid from parkinsonian patients. Neurosci Lett. 1994;165(1-2):208-210.

30. Murray KM, Dahl SL. Recombinant human tumor necrosis factor receptor (p75) Fc fusion protein (TNFR:Fc) in rheumatoid arthritis. Ann Pharmacother. 1997;31(11):1335-1338.

31. Boado RJ, Hui EK, Lu JZ, Zhou QH, Pardridge WM. Selective targeting of a TNFR decoy receptor pharmaceutical to the primate brain as a receptor-specific IgG fusion protein. J Biotechnol. 2010;146(1-2):84-91.

32. Grell M, et al. The transmembrane form of tumor necrosis factor is the prime activating ligand of the $80 \mathrm{kDa}$ tumor necrosis factor receptor. Cell. 1995;83(5):793-802.

33. Ruuls SR, et al. Membrane-bound TNF supports secondary lymphoid organ structure but is subservient to secreted TNF in driving autoimmune inflammation. Immunity. 2001;15(4):533-543.

34. Irvine KA, Blakemore WF. Age increases axon loss associated with primary demyelination in cuprizone-induced demyelination 
in C57BL/6 mice. J Neuroimmunol. 2006;175(1-2):69-76.

35. Marchetti L, Klein M, Schlett K, Pfizenmaier K, Eisel UL. Tumor necrosis factor (TNF)-mediated neuroprotection against glutamate-induced excitotoxicity is enhanced by N-methyl-D-aspartate receptor activation. Essential role of a TNF receptor 2-mediated phosphatidylinositol 3-kinase-dependent NF-kappa B pathway. J Biol Chem. 2004;279(31):32869-32881.

36. Remington LT, Babcock AA, Zehntner SP, Owens T. Microglial recruitment, activation, and proliferation in response to primary demyelination. Am J Pathol. 2007;170(5):1713-1724.

37. Muallem G, Hunter CA. ParadYm shift: Ym1 and Ym2 as innate immunological regulators of IL-17. Nat Immunol. 2014;15(12):1099-1100.

38. Mason JL, Suzuki K, Chaplin DD, Matsushima GK. Interleukin-1beta promotes repair of the CNS. J Neurosci. 2001;21(18):7046-7052.

39. Mason JL, Jones JJ, Taniike M, Morell P, Suzuki K, Matsushima GK. Mature oligodendrocyte apoptosis precedes IGF-1 production and oligodendrocyte progenitor accumulation and differentiation during demyelination/remyelination. J Neurosci Res. 2000;61(3):251-262

40. Ye JN, et al. Progesterone alleviates neural behavioral deficits and demyelination with reduced degeneration of oligodendroglial cells in cuprizone-induced mice. PLoS ONE. 2013;8(1):e54590.

41. Hesse A, et al. In toxic demyelination oligodendroglial cell death occurs early and is FAS independent. Neurobiol Dis. 2010;37(2):362-369

42. Goldberg J, et al. Short-term cuprizone feeding induces selective amino acid deprivation with concomitant activation of an integrated stress response in oligodendrocytes. Cell Mol Neurobiol. 2013;33(8):1087-1098.

43. Liñares D, et al. Neuronal nitric oxide synthase plays a key role in CNS demyelination. J Neurosci. 2006;26(49):12672-12681.

44. Kang Z, et al. IL-17-induced Act1-mediated signaling is critical for cuprizone-induced demyelination. J Neurosci. 2012;32(24):8284-8292.

45. McMahon EJ, Cook DN, Suzuki K, Matsushima GK. Absence of macrophage-inflammatory protein-1alpha delays central nervous system demyelination in the presence of an intact blood-brain barrier. J Immunol. 2001;167(5):2964-2971.

46. Taoufik E, et al. Transmembrane tumour necrosis factor is neuroprotective and regulates experimental autoimmune encephalomyelitis via neuronal nuclear factor-kappaB. Brain. 2011;134(Pt 9):2722-2735.

47. Maier O, Fischer R, Agresti C, Pfizenmaier K. TNF receptor 2 protects oligodendrocyte progenitor cells against oxidative stress. Biochem Biophys Res Commun. 2013;440(2):336-341.

48. Clausen $\mathrm{BH}$, et al. Systemically administered anti-TNF therapy ameliorates functional outcomes after focal cerebral ischemia. $J$ Neuroinflammation. 2014;11:203

49. Barnum CJ, et al. Peripheral administration of the selective inhibitor of soluble tumor necrosis factor (TNF) XPro®1595 attenuates nigral cell loss and glial activation in 6-OHDA hemiparkinsonian rats. J Parkinsons Dis. 2014;4(3):349-360.

50. Evangelidou M, Karamita M, Vamvakas SS, Szymkowski DE, Probert L. Altered expression of oligodendrocyte and neuronal marker genes predicts the clinical onset of autoimmune encephalomyelitis and indicates the effectiveness of multiple sclerosisdirected therapeutics. J Immunol. 2014;192(9):4122-4133.

51. Kotter MR, Zhao C, van Rooijen N, Franklin RJ. Macrophage-depletion induced impairment of experimental CNS remyelination is associated with a reduced oligodendrocyte progenitor cell response and altered growth factor expression. Neurobiol Dis. 2005;18(1):166-175.

52. Copelman CA, Diemel LT, Gveric D, Gregson NA, Cuzner ML. Myelin phagocytosis and remyelination of macrophageenriched central nervous system aggregate cultures. J Neurosci Res. 2001;66(6):1173-1178.

53. Ousman SS, David S. Lysophosphatidylcholine induces rapid recruitment and activation of macrophages in the adult mouse spinal cord. Glia. 2000;30(1):92-104.

54. Robinson S, Miller RH. Contact with central nervous system myelin inhibits oligodendrocyte progenitor maturation. Dev Biol. 1999;216(1):359-368.

55. Brück W, et al. Monocyte/macrophage differentiation in early multiple sclerosis lesions. Ann Neurol. 1995;38(5):788-796.

56. Prineas JW, Barnard RO, Kwon EE, Sharer LR, Cho ES. Multiple sclerosis: remyelination of nascent lesions. Ann Neurol. 1993;33(2):137-151

57. Kigerl KA, Gensel JC, Ankeny DP, Alexander JK, Donnelly DJ, Popovich PG. Identification of two distinct macrophage subsets with divergent effects causing either neurotoxicity or regeneration in the injured mouse spinal cord. J Neurosci. 2009;29(43):13435-13444.

58. Kroner A, Greenhalgh AD, Zarruk JG, Passos Dos Santos R, Gaestel M, David S. TNF and increased intracellular iron alter macrophage polarization to a detrimental M1 phenotype in the injured spinal cord. Neuron. 2014;83(5):1098-1116.

59. Pasparakis M, Alexopoulou L, Episkopou V, Kollias G. Immune and inflammatory responses in TNF alpha-deficient mice: a critical requirement for TNF alpha in the formation of primary B cell follicles, follicular dendritic cell networks and germinal centers, and in the maturation of the humoral immune response. J Exp Med. 1996;184(4):1397-1411.

60. Nicolussi EM, Huck S, Lassmann H, Bradl M. The cholinergic anti-inflammatory system limits T cell infiltration into the neurodegenerative CNS, but cannot counteract complex CNS inflammation. Neurobiol Dis. 2009;35(1):24-31.

61. Kielar C, Sawiak SJ, Navarro Negredo P, Tse DH, Morton AJ. Tensor-based morphometry and stereology reveal brain pathology in the complexin1 knockout mouse. PLoS One. 2012;7(2):e32636.

62. Xilouri M, Brekk OR, Polissidis A, Chrysanthou-Piterou M, Kloukina I, Stefanis L. Impairment of chaperone-mediated autophagy induces dopaminergic neurodegeneration in rats. Autophagy. 2016;12(11):2230-2247.

63. Reynolds ES. The use of lead citrate at high $\mathrm{pH}$ as an electron-opaque stain in electron microscopy. J Cell Biol. 1963;17:208-212

64. Kyrargyri V, Vega-Flores G, Gruart A, Delgado-García JM, Probert L. Differential contributions of microglial and neuronal IKK $\beta$ to synaptic plasticity and associative learning in alert behaving mice. Glia. 2015;63(4):549-566.

65. Nicole $\mathrm{O}$, et al. Neuroprotection mediated by glial cell line-derived neurotrophic factor: involvement of a reduction of NMDAinduced calcium influx by the mitogen-activated protein kinase pathway. J Neurosci. 2001;21(9):3024-3033.

66. Norton WT, Poduslo SE. Myelination in rat brain: method of myelin isolation. J Neurochem. 1973;21(4):749-757. 